O produto cartesiano de duas esferas mergulhado em uma esfera em codimensão um

Northon Canevari Leme Penteado 
SERVIÇO DE PÓS-GRADUAÇÃO DO ICMC-USP

Data de Depósito:

Assinatura:

\section{O produto cartesiano de duas esferas mergulhado em uma esfera em codimensão um}

\section{Northon Canevari Leme Penteado}

Orientador: Prof. Dr. Oziride Manzoli Neto

Dissertação apresentada ao Instituto de Ciências Matemáticas e de Computação - ICMC-USP, como parte dos requisitos para obtenção do título de Mestre em Ciências - Matemática. VERSÃO REVISADA.

USP - São Carlos

Março/2011 
Ficha catalográfica elaborada pela Biblioteca Prof. Achille Bassi e Seção Técnica de Informática, ICMC/USP, com os dados fornecidos pelo(a) autor(a)

Leme Penteado, Northon Canevari
O produto cartesiano de duas esferas mergulhado
em uma esfera em codimensão um / Northon Canevari
Leme Penteado; orientador Oziride Manzoli Neto --
São Carlos, 2011.
51 p.
Dissertação (Mestrado - Programa de Pós-Graduação en
Matemática) -- Instituto de Ciências Matemáticas e
de Computação, Universidade de São Paulo, 2011.
1. mergulho de variedades. 2. h-cobordismo. 3.
dualidades. 4. número interseção. 5. homologia e
cohomologia. I. Neto, Oziride Manzoli, orient. II.
Título.


"Talvez mais que teoricamente justos, esteticamente sensiveis ou politicamente inteligentes, o que nós precisamos ser é ativamente bons." José Saramago. 


\section{Agradecimentos}

A Deus pela vida e saúde.

Ao meu pai que com seu amor me proporcionou muitas alegrias e me ensinou a ter confiança na vida. À minha mãe que é o meu maior exemplo de perseverança e dedicação à família e a minha querida irmã que apesar de tudo sempre está do meu lado. Agradeço também aos meus avós que sempre me deram apoio e juízo. E não podia faltar a família que sempre me recebeu com muito carinho, que é a família da minha namorada e é claro, minha namorada Cristiane Rodrigues por ser uma extraordinária companheira e amiga que sempre esteve ao meu lado nos melhores e piores momentos, me fazendo sentir vivo.

Ao meu orientador Prof. Dr. Oziride Manzoli Neto (Dide), pela sincera amizade, pelos conselhos, pela sua contagiante paixão a matemática, pelo seu exemplo de dedicação ao trabalho e pelo sua constante disposição em ajudar nos problemas surgidos no decorrer da dissertação.

Aos meus companheiros de toda hora Alex, Deliene, Diego, Edson, Gustavo, Isac, Javier, Rafael D., Rafael R., Murilo, Tais. Aos amigos e colegas de estudos, pelos estudos em grupos e por tudo que sorrimos e sofremos juntos, tanto os de graduação quanto os de mestrado. Em particular Alex, Andreza, Apoenã, Henry, Nelson, Rafael, Renato, Thaís, Vinicius e um grande amigo e excelente companheiro de estudo Sérgio. E também aos amigos de repúblicas.

Ao Instituto de Ciências Matemáticas e de Computação pela colaboração e excelente 
estrutura para a pós graduação. Agradeço também ao CNPq pela concessão da bolsa. 


\section{Resumo}

James W. Alexander, no artigo [1], mostra que se tivermos um mergulho PL $f$ : $S^{1} \times S^{1} \rightarrow S^{3}$, então o fecho de uma das componentes conexas de $S^{3}-f\left(S^{1} \times S^{1}\right)$ é homeomorfo a um toro sólido, isto é, homeomorfo a $S^{1} \times D^{2}$. Este teorema ficou conhecido por Teorema do toro de Alexander. Nesta dissertação, estamos detalhando a demonstração deste teorema feita em [25] que é diferente da demonstração apresentada em [1. Mais geralmente, para um mergulho diferenciável $f: S^{p} \times S^{q} \rightarrow S^{p+q+1}$, demonstra-se que o fecho de uma das componentes conexas de $S^{p+q+1}-f\left(S^{p} \times S^{q}\right)$ é difeomorfo a $S^{p} \times D^{q+1}$ se $p \geq q \geq 1$ e $p+q \neq 3$ ou se $p=2$ e $q=1$ um dos fechos será homeomorfo a $S^{2} \times D^{2}$, nesta dissertação estaremos também detalhando estas demonstrações feita em [20]. 


\section{Abstract}

James W. Alexander shows in [1] that the closure of one of the two connected components of $S^{3}-f\left(S^{1} \times S^{1}\right)$ is homeomorphic to a solid torus $S^{1} \times D^{2}$, where $f: S^{1} \times S^{1} \rightarrow S^{3}$ is a PL embedding. This result became known as Alexander's torus theorem. In this dissertation we are detailing the proof of this theorem made in [25] which is different from the demonstration presented in [1]. More generally, when considering a smooth embeding $f: S^{p} \times S^{q} \rightarrow S^{p+q+1}$, it is demonstrated that the closure of one of the two connected components $S^{p+q+1}-f\left(S^{p} \times S^{q}\right)$ is diffeomorphic to $S^{p} \times D^{q+1}$ if $p \geq q \geq 1$ and $p+q \neq 3$ or if $p=2$ and $q=1$ one of the closures will be homeomorphic to $S^{2} \times D^{2}$. In this work we are also detailing the proves made in [20]. 



\section{Sumário}

Introdução

1 Pré-requisitos 3

1.1 Teorema de van Kampen . . . . . . . . . . . . . . . . . . . . . . . 3

1.2 Os Teoremas de Hurewicz . . . . . . . . . . . . . . . . . . . . . 4

1.3 O Teorema de Whitehead ............... 5

1.4 Produtos . . . . . . . . . . . . . . . . . . . . . . 6

1.4.1 Produto Tensor e Resultados . . . . . . . . . . . . . . . . . . . 6

1.4.2 Produto Cup e Produto Cap . . . . . . . . . . . . . . . . 8

1.4 .3 Produto $E x t \ldots \ldots \ldots \ldots \ldots$

1.5 Homologia e Cohomologia para Variedades Diferenciáveis . . . . . . . . . . 11

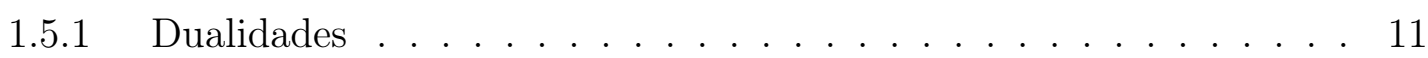

1.5 .2 Teorema do h-Cobordismo . . . . . . . . . . . . . . 11

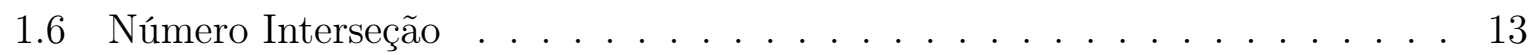

$\begin{array}{lll}2 & \text { Mergulhos de produto de esferas em esferas } & 17\end{array}$

2.1 Mergulhos PL do Toro em $S^{3} \ldots \ldots \ldots \ldots \ldots$. . . . . . . . . . 17

2.2 Mergulhos de $S^{p} \times S^{q}$ em $S^{p+q+1} \ldots \ldots \ldots \ldots \ldots \ldots . \ldots \ldots$

2.2 .1 Caso em que $p>q>2 \ldots \ldots \ldots \ldots \ldots$

2.2 .2 Caso em que $p=q \geq 2 \ldots \ldots \ldots \ldots$. . . . . . . . . . . 27 
2.2 .3 Caso em que $p>q=1 \ldots \ldots \ldots \ldots$ 


\section{Introdução}

Essencialmente este trabalho trata de mergulhos de produtos de esferas em esferas de forma que a codimensão seja um. Na topologia, quando se considera mergulhos, temos três principais problemas:

(a) Determinar se existe mergulhos de um espaço em outro;

(b) se existir, classificar, usando difeomorfismo ou algum invariante, os tipos de mergulhos que existem;

(c) caracterizar quando estes mergulhos são triviais.

Inicialmente pensou-se em mergulhos de $S^{1}$ em outros espaço, o que deu origem à Teoria dos Nós. Seguindo nessa linha, James W. Alexander, em [1], classificou os mergulhos PL (piecewise linear) de $S^{1} \times S^{1}$ em $S^{3}$, mostrando que considerando um mergulho PL $f: S^{1} \times S^{1} \rightarrow S^{3}$, então o fecho de umas das componentes conexas de $S^{3}-f\left(S^{1} \times S^{1}\right)$ borda um toro sólido $S^{1} \times D^{2}$ e este resultado ficou conhecido como Teorema do toro de Alexander. Com este resultado, gerou-se vários trabalhos sobre generalizações. Por exemplo, sob algumas condições para $p$ e $q$, considerou-se problemas de classificações de mergulhos de $S^{p} \times S^{q}$ em $S^{p+q+1}$ nos trabalhos [13], [9] e [20], entre outros. Este texto é sobre a classificação de mergulhos deste tipo. Dado um mergulho diferenciável $f: S^{p} \times S^{q} \rightarrow S^{p+q+1}$, estuda-se o fecho das componentes conexas de $S^{p+q+1}-f\left(S^{p} \times S^{q}\right)$.

Basicamente este trabalho se divide em dois capítulos. O primeiro capítulo, intitulado Pré-requisitos, é dedicado às definições e resultados necessários para o desenvolvimento e compreensão do texto. Para os leitores que tenham conhecimentos do conteúdo deste capítulo, ele será útil para fixarmos notações e terminologia. 
Para classificar os mergulhos de $S^{p} \times S^{q}$ em $S^{p+q+1}$ divide-se em casos dependendo de $p$ e $q$. No segundo capítulo trata no caso $p=q=1$, que é o teorema do toro de Alexander, de uma forma diferente de [1].

Neste mesmo capítulo, caracteriza-se os mergulhos de $S^{p} \times S^{q}$ em $S^{p+q+1}$ para os outros casos de $p$ e $q$. A caracterização é da seguinte forma; dado um mergulho suave $f: S^{p} \times S^{q} \rightarrow S^{p+q+1}$, então um dos fechos das componentes conexas de $S^{p+q+1}-f\left(S^{p} \times S^{q}\right)$ é difeomorfo a $S^{p} \times D^{q+1}$ se $p+q \neq 3$ e se $p+q=3$ então um dos fechos das componentes conexas e homeomorfo a $S^{2} \times D^{2}$. 


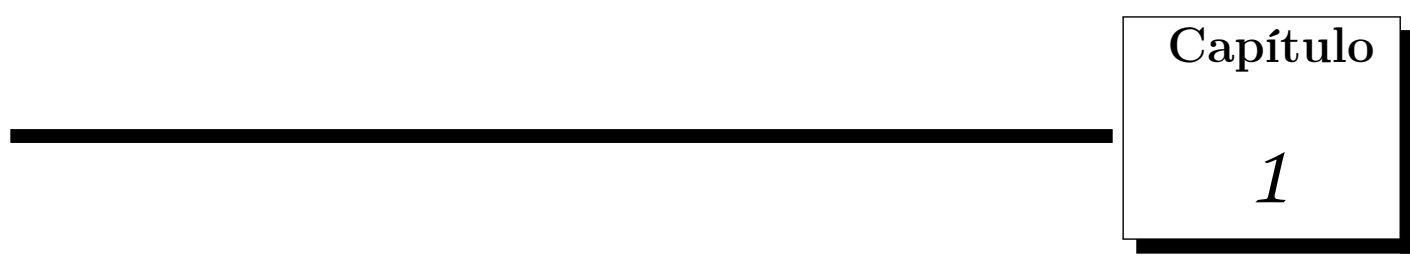

\section{Pré-requisitos}

\subsection{Teorema de van Kampen}

Seja um espaço topológico $X$ tal que $U_{1} \cup U_{2}=X$, onde $U_{1}, U_{2}$ e $U_{1} \cap U_{2}$ são conexo por caminhos com $U_{1} \cap U_{2} \neq \emptyset$. Denotaremos $i_{1}, i_{2}, j_{1}$ e $j_{2}$ as inclusões que comutam o diagrama abaixo

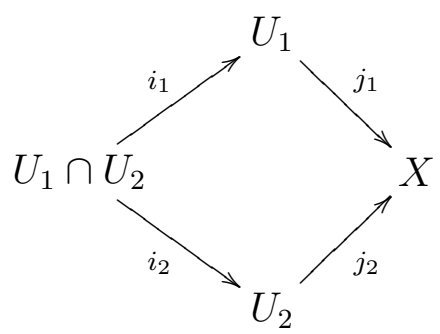

Então o diagrama de homomorfismos abaixo também é comutativo.

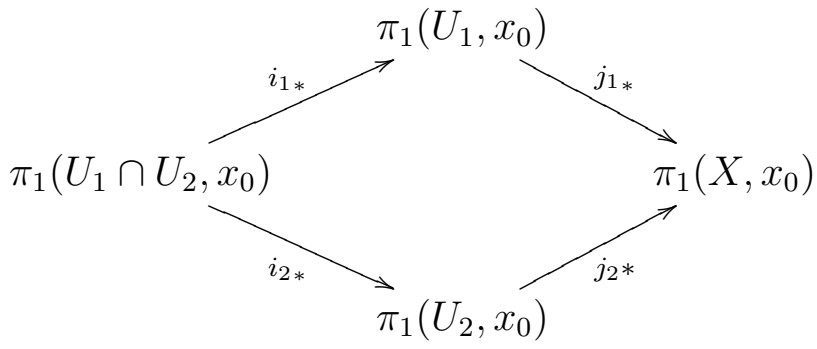

Suponhamos que conhecemos $\pi_{1}\left(U_{1}, x_{0}\right), \pi_{1}\left(U_{2}, x_{0}\right), \pi_{1}\left(U_{1} \cap U_{2}, x_{0}\right)$ e suas apresentações 
são dadas por:

$$
\begin{array}{ll}
\pi_{1}\left(U_{1}, x_{0}\right) & =<S_{1} ; R_{1}> \\
\pi_{1}\left(U_{2}, x_{0}\right) & =<S_{2} ; R_{2}> \\
\pi_{1}\left(U_{1} \cap U_{2}, x_{0}\right) & =<S ; R>
\end{array}
$$

onde $x_{0} \in U_{1} \cap U_{2}$ é fixado, $S, S_{1}$ e $S_{2}$ são os geradores e $R, R_{1}$ e $R_{2}$ são as relações dos grupos acima. Lembrando que $<G ; R>$ é a notação para o grupo com o conjunto $G$ como geradores satisfazendo as relações de $R$.

Definamos um conjunto de relações sobre o conjunto $S_{1} \cup S_{2}$ da seguinte forma $R_{S}=$ $\left\{i_{1 *}(s)=i_{2 *}(s) ; \forall s \in S\right\}$. Dessa forma temos o teorema:

Teorema 1.1.1 (Teorema de van Kampen). Dado um espaço topológico $X$ tal que $U_{1} \cup$ $U_{2}=X$, onde $U_{1}, U_{2}$ e $U_{1} \cap U_{2}$ são conexos por caminhos com $U_{1} \cap U_{2} \neq \emptyset$ e $x_{0} \in U_{1} \cap U_{2}$ fixo. Então $\pi_{1}\left(X, x_{0}\right)$ é isomorfo ao grupo definido pelos geradores $S_{1} \cup S_{2}$ e as relações $R_{1} \cup R_{2} \cup R_{S}$.

Corolário 1.1.1.1. Suponha que $X$ é um espaço topológico tal que $U_{1} \cup U_{2}=X$, onde $U_{1}, U_{2}$ e $U_{1} \cap U_{2}=T$ são conexo por caminhos com $T \neq \emptyset$ e $x_{0} \in T$ fixo. Consideremos $i_{1}: T \rightarrow X_{1}, i_{2}: T \rightarrow X_{2}, j_{1}: X_{1} \rightarrow X$ e $j_{2}: X_{2} \rightarrow X$ as inclusões. Se $i_{1 *}$ e $i_{2 *}$ forem injetivas então $j_{1 *}$ e $j_{2 *}$ também o são.

\subsection{Os Teoremas de Hurewicz}

O matemático Witold Hurewicz, no século XX, demonstrou resultados muito importantes, os quais relacionam os grupos de homologia com os grupos de homotopia. Alguns resultados são:

Teorema 1.2.1 (Teorema do Homomorfismo de Hurewicz). Consideremos dois espaços topológicos com seus respectivos pontos base $\left(X, x_{0}\right)$ e $\left(Y, y_{0}\right)$, um subespaço $A$ de $X$ contendo $x_{0}$, um subespaço $B$ de $Y$ contendo $y_{0}$ e uma aplicação $f:\left(X, A, x_{0}\right) \rightarrow\left(Y, B, y_{0}\right)$. Se $n \geq 2$ ou se $n=1$ e $A=\left\{x_{0}\right\}$, então existem homomorfismos $\varphi_{1}: \pi_{n}\left(X, A, x_{0}\right) \rightarrow$ 
$H_{n}(X, A)$ e $\varphi_{2}: \pi_{n}\left(Y, B, y_{0}\right) \rightarrow H_{n}(Y, B)$ tal que comuta o diagrama

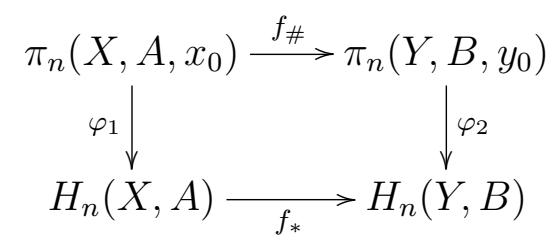

Teorema 1.2.2. Se X é um espaço conexo por caminho, então o homomorfismo de Hurewicz $\varphi: \pi_{1}(X) \rightarrow H_{1}(X)$ é sobrejetor e seu núcleo é o subgrupo comutador de $\pi_{1}(X)$. Portanto $H_{1}(X)$ é isomorfo a $\frac{\pi_{1}(X)}{\left[\pi_{1}(X)\right]}$.

Teorema 1.2.3 (Teorema de Hurewicz). Seja X um espaço topológico simplesmente conexo. Então as seguintes afirmações são equivalentes:

- $\pi_{j}(X)=1$ para todo $j=1, \ldots, n-1$

- $H_{j}(X)=0$ para todo $j=1, \ldots, n-1$.

Além disso, no caso em que um dos dois item for satisfeito, segue que $H_{n}(X)$ é isomorfo $a \pi_{n}(X)$.

As provas destes teoremas e outros resultados podem serem encontrados no livro [29] nas Seções 4 e 5 no Capítulo 7.

\subsection{O Teorema de Whitehead}

Definição 1.3.1. Sejam $X$ e $Y$ espaços topológicos e $f: X \rightarrow Y$ uma aplicação contínua. Dizemos que f é uma equivalência de homotopia fraca se $f$ induz uma correspondência biunívoca entre as componentes conexas por caminhos de $X$ e as componentes conexas por caminhos de $Y$ e se para todo $n \geq 1$ e $x \in X$ a induzida em homotopia $f_{\#}: \pi_{n}(X, x) \rightarrow$ $\pi_{n}(Y, f(x))$ é um isomorfismo.

Observemos que se $f: X \rightarrow Y$ é uma equivalência de homotopia, então $f$ é uma equivalência de homotopia fraca. Além disso, pelo Corolário 24 de ([29], pag 405) se $X$ e $Y$ forem variedades suaves $\left(C^{\infty}\right)$, então ambas têm estrutura de complexos $\mathrm{CW}$ e os conceitos de equivalência de homotopia e equivalência de homotopia fraca coincidem. 
Considerando este resultado junto com o Teorema 7.15 de ([34], pag 182), temos o seguinte teorema:

Teorema 1.3.1 (Teorema de Whitehead). Sejam $X$ e $Y$ complexos $C W$ finitos, simplesmente conexos e $f: X \rightarrow Y$ uma função contínua tal que para todo n inteiro não negativo, $f_{*}: H_{n}(X) \rightarrow H_{n}(Y)$ é isomorfismo. Então $f$ é uma equivalênncia de homotopia.

\subsection{Produtos}

\subsubsection{Produto Tensor e Resultados}

Sejam $A, B$ grupos. Consideremos $A \times B$ e $F(A \times B)$ o grupo livre gerado por $A \times B$ onde os elementos são dados por

$$
\sum n_{i}\left(a_{i}, b_{i}\right)
$$

onde $n_{i} \in \mathbb{Z}$ e a é soma finita. Tomemos o subgrupo de $F(A \times B)$, denotado por $R(A \times B)$, gerado pelos elementos da forma

$$
\left(a_{1}+a_{2}, b\right)-\left(a_{1}, b\right)-\left(a_{2}, b\right)
$$

e

$$
\left(a, b_{1}+b_{2}\right)-\left(a, b_{1}\right)-\left(a, b_{2}\right)
$$

Definição 1.4.1. O produto tensor de $A$ e $B$ é definido por

$$
A \otimes B=\frac{F(A \times B)}{R(A \times B)}
$$

Proposição 1.4.1. 1) Dados grupos $A$ e $B$, então $A \otimes B \approx B \otimes A$.

2) Se $A \approx \sum A_{j}$ então $A \otimes B \approx \sum\left(A_{j} \otimes B\right)$.

3) Para todo grupo abeliano $A, \mathbb{Z} \otimes A \approx A$.

4) Sejam $i_{1}: A^{\prime} \rightarrow A$ e $i_{2}: B^{\prime} \rightarrow B$ inclusões, então

$$
\frac{A}{A^{\prime}} \otimes \frac{B}{B^{\prime}} \approx \frac{A \otimes B}{\operatorname{Im}\left(i_{1} \otimes i d\right)+\operatorname{Im}\left(i d \otimes i_{2}\right)} .
$$


5) Se a sequência $A \stackrel{\alpha}{\longrightarrow} B \stackrel{\beta}{\longrightarrow} C \longrightarrow 0$ é exata então para qualquer grupo abeliano $D$ temos a sequência

$$
A \otimes D \stackrel{\alpha \otimes i d}{\longrightarrow} B \otimes D \stackrel{\beta \otimes i d}{\longrightarrow} C \otimes D \longrightarrow 0
$$

que é exata.

As provas destes itens se encontra em ([31], cap 3).

Uma consequência imediata do item 4 é que o grupo $\mathbb{Z}_{q} \otimes \mathbb{Z}_{p}$ é isomorfo a $\mathbb{Z}_{(q, p)}$, onde $(q, p)$ é o máximo divisor comum entre $p$ e $q$.

Definição 1.4.2. Uma resolução livre de um grupo A é uma sequência exata curta

$$
0 \longrightarrow G_{1} \stackrel{j}{\longrightarrow} G_{0} \stackrel{\tau}{\longrightarrow} A \longrightarrow 0
$$

onde $G_{0}$ e $G_{1}$ são grupos abelianos livres.

Definição 1.4.3. Dados uma resolução livre de $A$ e um grupo abeliano D. Pelo item 5 da proposição 1.4.1 a sequência

$$
G_{1} \otimes D \stackrel{j \otimes i d}{\longrightarrow} G_{0} \otimes D \stackrel{\tau \otimes i d}{\longrightarrow} A \otimes D \longrightarrow 0
$$

é exata. Portanto definimos $\operatorname{Tor}(A, D)=\operatorname{ker}(j \otimes i d)$.

Os próximos resultados podem ser encontrados em [29] página 243 e página 230, respectivamente.

Teorema 1.4.1 (Teorema dos Coeficientes Universais para Homologia). Se C é um complexo de cadeias livre e Gé um grupo abeliano, então

$$
H_{n}(C \otimes G) \approx H_{n}(C) \otimes G \oplus \operatorname{Tor}\left(H_{n-1}(C), G\right)
$$

Teorema 1.4.2 (Fórmula de Küneth). Sejam X e Y são espaços topológicos, então

$$
H_{n}(X \times Y) \approx \sum_{p+q=n} H_{p}(X) \otimes H_{q}(Y) \oplus \sum_{r+s=n-1} \operatorname{Tor}\left(H_{r}(X), H_{s}(Y)\right)
$$




\subsubsection{Produto Cup e Produto Cap}

Para definir produto cup e produto cap precisaremos de algumas observações e definições antes.

Dado um complexo de cadeia $\mathcal{C}=\left(C_{q}, \partial_{q}\right)$ podemos considerar o complexo de cocadeia dual de $\mathcal{C}$, denotado por $\mathcal{C}^{*}=\left(C^{q}, \delta^{q}\right)$, onde $C^{q}=\operatorname{Hom}\left(C^{q}, \mathbb{Z}\right)$ e $\delta^{q}: C^{q} \rightarrow C^{q-1}$ é definido por $\delta^{q}(h)=(-1)^{q+1} h \circ \partial_{q+1}^{*} \in C^{q+1}$ para $h \in C^{q}$.

Definição 1.4.4. Se $\mathcal{C}$ e $\mathcal{C}^{\prime}$ são complexos de cadeias, definimos $\mathcal{C} \otimes \mathcal{C}^{\prime}$ por $\left(C \otimes C^{\prime}\right)_{n}=$ $\bigoplus_{i+j=n} C_{i} \otimes C^{\prime}{ }_{j}$ e $\partial \otimes \partial^{\prime}\left(c \otimes c^{\prime}\right)=\partial c \otimes c^{\prime}+(-1)^{k} c \otimes \partial^{\prime} c^{\prime}$, se $c \in C_{k}$. Assim podemos definir o operador slant

$$
/:\left(C \otimes C^{\prime}\right)_{n} \otimes C^{\prime k} \rightarrow C_{n-k}
$$

por $a / b=\sum_{i+j=n} b\left(a_{j}^{\prime}\right) a_{i}$, onde $a=\bigoplus_{i+j=n}\left(a_{i} \otimes a^{\prime}{ }_{j}\right)$ e $b\left(a^{\prime}{ }_{j}\right)=0$ sempre que $j \neq k$.

Para ver que esta operação está bem definida com imagem em $C_{n-k}$, note que primeiramente sendo $b \in C^{\prime k}=\operatorname{Hom}\left(C^{\prime}{ }_{k}, \mathbb{Z}\right)$, temos $b$ um homomorfismo $b: C^{\prime}{ }_{k} \rightarrow \mathbb{Z}$. Além disso, definimos $b\left(a_{j}^{\prime}\right)=0$ se $j \neq k$. Mas quando $j=k$, com $k$ fixo, nas somas que definem o operador slant temos $i=n-k$. Logo

$$
a / b=\sum_{i+j=n} b\left(a_{j}^{\prime}\right) a_{i}=b\left(a_{k}^{\prime}\right) a_{n-k} \in C_{n-k}
$$

Em [4] é mostrado que o operador slant é uma aplicação de cadeia e, portanto, podese induzir uma aplicação $H_{*}\left(\left(\mathcal{C} \otimes \mathcal{C}^{\prime}\right) \otimes C^{* *}\right) \rightarrow H_{*}(\mathcal{C})$. Compondo esta com a aplicação natural

$$
H_{*}\left(\mathcal{C} \otimes \mathcal{C}^{\prime}\right) \otimes H^{*}\left(\mathcal{C}^{\prime}\right) \rightarrow H_{*}\left(\left(\mathcal{C} \otimes \mathcal{C}^{\prime}\right) \otimes \mathcal{C}^{\prime *}\right)
$$

chegamos ao operador slant

$$
/: H_{n}\left(\mathcal{C} \otimes \mathcal{C}^{\prime}\right) \otimes H^{k}\left(\mathcal{C}^{\prime}\right) \rightarrow H_{n-k}(\mathcal{C})
$$

Agora, seja $\mathcal{C}$ um complexo de cadeias aumentado com uma aplicação diagonal, dig$\operatorname{amos} \Delta: \mathcal{C} \rightarrow \mathcal{C} \otimes \mathcal{C}$ tal que $(\epsilon \otimes 1) \Delta(c)=c$, onde $\epsilon: \mathcal{C} \rightarrow \mathbb{Z}$ é o aumento, e identificamos 
$\mathcal{C}=\mathcal{C} \otimes \mathbb{Z}=\mathbb{Z} \otimes \mathcal{C}$. Então $\Delta$ induz em $\mathcal{C}^{*}$ a estrutura de um anel com unidade por $\mathcal{C}^{*} \otimes \mathcal{C}^{*} \rightarrow(\mathcal{C} \otimes \mathcal{C})^{*} \stackrel{\Delta^{*}}{\rightarrow} \mathcal{C}^{*}$, onde $\mathcal{C}^{*} \otimes \mathcal{C}^{*} \rightarrow(\mathcal{C} \otimes \mathcal{C})^{*}$ é a inclusão.

Definição 1.4.5. Esta construção feita acima é chamado de produto cup, e em nível de cohomologia induz

$$
\smile: H^{n}(\mathcal{C}) \otimes H^{k}(\mathcal{C}) \rightarrow H^{n+k}(\mathcal{C})
$$

o qual também é chamado de produto cup.

Definição 1.4.6. Consideremos $\Delta$ como acima. Definimos o produto cap

$$
\frown: C_{n} \otimes C^{k} \rightarrow C_{n-k}
$$

pela fórmula $a \frown b:(\Delta a) / b$. Como $\frown$ é a composição das aplicações de cadeias $\Delta e /$, segue que $\frown$ é uma aplicação de cadeia e induz

$$
\frown: H_{n}(\mathcal{C}) \otimes H^{k}(\mathcal{C}) \rightarrow H_{n-k}(\mathcal{C})
$$

Mais geralmente, suponha que $\mathcal{A}, \mathcal{B}$ e $\mathcal{C}$ sejam complexos de cadeias aumentados e seja $\Delta: \mathcal{A} \rightarrow \mathcal{B} \otimes \mathcal{C}$ uma aplicação de cadeias. Então similarmente ao anterior, obtemos uma aplicação em cohomologia

$$
\smile: H^{p}(\mathcal{B}) \otimes H^{q}(\mathcal{C}) \rightarrow H^{p+q}(\mathcal{A})
$$

e o produto cap

$$
\frown: H_{n}(\mathcal{A}) \otimes H^{k}(\mathcal{C}) \rightarrow H_{n-k}(\mathcal{B})
$$

Para que se possa ter uma melhor compreensão desta seção, pode-se encontrar outros resultados sobre produto cup e produto cap por exemplo, em [4] e [8].

\subsubsection{Produto Ext}

O produto Exté importante e um dos resultados é o Teorema do Coeficientes Universais para Cohomologia. As demonstrações dos resultados a seguir são encontradas em [31] 
Proposição 1.4.2. Se $0 \rightarrow F \stackrel{i}{\rightarrow} H \rightarrow \stackrel{\pi}{\rightarrow} K \rightarrow 0$ é uma sequência exata que cinde onde $F, H$ e $K$ são grupos abelianos e dado $G$ um grupo abeliano, então

$$
0 \rightarrow \operatorname{Hom}(K, G) \stackrel{\pi^{*}}{\rightarrow} \operatorname{Hom}(H, G) \stackrel{i^{*}}{\rightarrow} \operatorname{Hom}(F, G) \rightarrow 0
$$

é exata.

A demonstração pode ser encontrada em ([31], pag 79).

Definição 1.4.7. Dado $f: G \rightarrow H$ um homomorfismo. O núcleo de $f$ é o subgrupo ker $f=f^{-1}(0)$, a imagem de $f$ é o subgrupo $f(G)=\operatorname{Im} f$ e o conúcleo de $f$ é o grupo quociente coker $f=\frac{H}{\operatorname{Imf}}$.

Observação: Seja E um grupo abeliano e tomemos uma resolução livre de E,

$$
0 \rightarrow R \stackrel{i}{\rightarrow} F \stackrel{\pi}{\rightarrow} E \rightarrow 0
$$

isto é, a sequência acima é exata curta e $R, F$ são abelianos livres.

Dado qualquer grupo abeliano $G$ a sequência

$$
0 \rightarrow \operatorname{Hom}(E, G) \stackrel{\pi^{*}}{\rightarrow} \operatorname{Hom}(F, G) \stackrel{i^{*}}{\rightarrow} \operatorname{Hom}(R, G)
$$

é exata pela proposição 1.4.2.

Definição 1.4.8. $\operatorname{Ext}(E, G)=\operatorname{coker} i^{*}=\frac{\operatorname{Hom}(R, G)}{\operatorname{Im} i^{*}}$.

Uma propriedade interessante é que quando $E$ é um grupo abeliano livre então $\operatorname{Ext}(E, G)=$ 0.

O próximo resultado pode ser encontrado em [29] página 222.

Teorema 1.4.3 (Teorema dos Coeficientes Universais para Cohomologia). Se Cé um complexo de cadeia livre e $G$ um grupo abeliano, então

$$
H^{q}(C, G) \approx \operatorname{Hom}\left(H_{q}(C), G\right) \oplus \operatorname{Ext}\left(H_{q-1}(C), G\right) .
$$




\subsection{Homologia e Cohomologia para Variedades Diferen- ciáveis}

\subsubsection{Dualidades}

As dualidades são interessantes pelo fato de que elas relacionam homologia com cohomologia. Os próximos resultados podem ser encontrados em ([24], pag 419), ([11], pag 254) e ([24], pag 424). Usaremos o conceito de homologia reduzida que pode ser encontrada em 24] página 43.

Teorema 1.5.1 (Dualidade de Poincaré). Dado uma n-variedade $M$ compacta, conexa, sem bordo e com orientação $s: M \rightarrow F$ e associada a classe fundamental z, então o homomorfismo

$$
D: H^{k}(M ; G) \rightarrow H_{k}(M ; G)
$$

dado por $D(x)=x \cap z$, é um isomorfismo para cada $k \in \mathbb{Z}^{+}$.

Teorema 1.5.2 (Dualidade de Poincaré-Lefschetz). Seja $M$ uma n-variedade com bordo $\partial M . S e(M, \partial M)$ é orientável, então

$$
H^{p}(M, \partial M) \approx H_{n-p}(M) \text { e } H^{p}(M) \approx H_{n-p}(M, \partial M) .
$$

Teorema 1.5.3 (Dualidade de Alexander). Seja A um subconjunto próprio não vazio da esfera $S^{n}$. Suponha que A seja um retrato de alguma vizinhança. Então

$$
\tilde{H}^{n-k}(A) \approx \tilde{H}_{k-1}\left(S^{n}-A\right)
$$

onde $\tilde{H}^{*}(A)$ e $\tilde{H}_{*}\left(S^{n}-A\right)$ são as homologias reduzidas de $A$ e $S^{n}-A$.

\subsubsection{Teorema do h-Cobordismo}

Os próximos resultados podem ser encontrados em [23]. 
Definição 1.5.1. Uma terna $\left(W ; V_{0}, V_{1}\right)$ é uma tríade de variedades suaves se $W$ for uma n-variedade suave compacta e o bordo de $W$ é a união disjunta de duas subvariedades sem bordo $V_{0}$ e $V_{1}$.

Definição 1.5.2. Dadas duas variedades compactas e sem bordos $M_{0}$ e $M_{1}$. Um cobordismo de $M_{0}$ a $M_{1}$ é uma 5-upla $\left(W ; V_{0}, V_{1} ; h_{0}, h_{1}\right)$ onde $\left(W ; V_{0}, V_{1}\right)$ é uma tríade de variedades suaves e $h_{i}: V_{i} \rightarrow M_{i} i=1,2$ são difeomorfismo.

A prova dos próximos dois resultados estão em ([23], pag 23).

Teorema 1.5.4 (Teorema da Vizinhança Colarinho). Seja W uma variedade compacta suave com bordo $B d(W)$ diferente de vazio. Existe uma vizinhança de $B d(W)$, chamada vizinhança colarinho, difeomorfa a $B d(W) \times[0,1)$

Definição 1.5.3. Uma subvariedade conexa e fechada $M^{n-1}$ de $W^{n}-B d\left(W^{n}\right)$ é dita ser 2-lados se alguma vizinhança de $M^{n-1}$ em $W^{n}$ é dividida em duas componentes quando $M^{n-1}$ é deletada.

Teorema 1.5.5 (Teorema do Bicolarinho). Suponha que toda componente de uma subvariedade $M$ de $W$ é compacta e 2-lados. Então existe uma vizinhança, chamada vizinhança bicolar, de $M$ em $W$ difeomorfa a $M \times(-1,1)$ tal que $M$ corresponde a $M \times 0$.

Para os dois teoremas abaixo ver livro 23] páginas 107 e 109, respectivamente.

Teorema 1.5.6 (Teorema do h-Cobordismo). Suponha $W$ uma n-variedade compacta e $\left(W ; V_{0}, V_{1}\right)$ é uma tríade de variedades com as as seguintes prorpiedades:

1) $W, V_{0}$ e $V_{1}$ são simplesmente conexas.

2) $H_{*}\left(W, V_{0}\right)=0$

3) $\operatorname{dim} W=n \geq 6$.

Então $W$ é difeomorfa a $V_{0} \times[0,1]$.

Teorema 1.5.7 (Conjectura de Poincaré Generalizada). Se M é uma n-variedade fechada, conexa, simplesmente conexa e $n \geq 4$. Então $M$ é homeomorfa a esfera $S^{n}$. 


\subsection{Número Interseção}

O número interseção de dois espaços $A, B$ (normalmente variedades) mergulhados num ambiente $C$ (também variedade) cuja dimensão é a soma das dimensões dos espaços $A$ e $B$ "mede o tamanho" da interseção mínima entre mergulhos transversais isotópicos de $A$ e $B$ em $C$. Em [8] é feito somente número interseção para variedades transversais.

Tanto o número interseção em $\mathbb{R}^{n}$ quanto em variedades podem ser encontrados em [6. Assim as provas dos resultados apresentados aqui podem serem encontradas em tal livro.

Definição 1.6.1. Sejam $A \subset X \subset \mathbb{R}^{n}, B \subset Y \subset \mathbb{R}^{n}$ tal que $A \cap Y=X \cap B=\emptyset$ e considere a função $d:(X \times Y, A \times Y \cup X \times B) \rightarrow\left(\mathbb{R}^{n}, \mathbb{R}^{n}-\{0\}\right)$ dada por $d(x, y)=x-y$.

A decomposição

$$
H_{n-i}(X, A) \times H_{i}(Y, B) \stackrel{\times}{\rightarrow} H_{n}(X \times Y, A \times Y \cup X \times B) \rightarrow
$$

$\stackrel{(-1)^{i} d_{*}}{\longrightarrow} H_{n}\left(\mathbb{R}^{n}, \mathbb{R}^{n}-\{0\}\right)$

é chamada a interseção de pares. Escrevemos $\xi \circ \eta=(-1)^{i} d_{*}(\xi \times \eta)$, para $\xi \in$ $H_{n-i}(X, A)$ e $\eta \in H_{i}(Y, B)$, o qual é um elemento de $H_{n}\left(\mathbb{R}^{n}, \mathbb{R}^{n}-\{0\}\right) \approx \mathbb{Z}$ e é chamado número interseção de $\xi$ e $\eta$.

Proposição 1.6.1. Se $X \cap Y=\emptyset$ então toda interseção de pares $H_{n-i}(X, A) \times H_{i}(Y, B) \rightarrow$ $H_{n}\left(\mathbb{R}^{n}, \mathbb{R}^{n}-\{0\}\right)$ é nula.

Proposição 1.6.2. Dados uma interseção de pares $H_{n-i}(X, A) \times H_{i}(Y, B) \rightarrow H_{n}\left(\mathbb{R}^{n}, \mathbb{R}^{n}-\right.$ $\{0\}), \xi \in H_{n-i}(X, A)$ e $\eta \in H_{i}(Y, B)$. Ent $\tilde{a} \circ \xi \circ \eta=(-1)^{i(n-i)} \eta \circ \xi$.

Neste caso é definido número interseção sobre variedades.

Definição 1.6.2. Dada uma função $f: M^{\prime m^{\prime}} \rightarrow M^{m}$ entre duas variedades. A função transfer de $f$, denotada por $f_{!}: H_{j}(V, U) \rightarrow H_{j+\left(m^{\prime}-m\right)}\left(f^{-1}(V), f^{-1}(U)\right)$, é definida pela seguinte composição

$$
\begin{aligned}
& \quad H_{j}(V, U) \stackrel{(\frown o)^{-1}}{\longrightarrow} \check{H}_{c}^{m-j}(M-U, M-V) \stackrel{\check{f}_{c}}{\longrightarrow} \check{H}_{c}^{m-j}\left(M^{\prime}-f^{-1}(U), M^{\prime}-f^{-1}(V)\right) \stackrel{\frown o^{\prime}}{\longrightarrow} \\
& H_{m^{\prime}-m+j}\left(f^{-1}(V), f^{-1}(U)\right) .
\end{aligned}
$$


Definição 1.6.3. Sejam $M^{n}$ uma variedade orientada e $d: M \rightarrow M \times M$ o mergulho diagonal, isto é, $d(x)=(x, x)$. Consideremos, para os pares de abertos $(V, S)$ e $(W, T)$ de $M$, a função;

$$
H_{i}(V, S) \otimes H_{j}(W, T) \stackrel{\times}{\rightarrow} H_{i+j}(V \times W, S \times W \cup V \times T) \rightarrow
$$

$H_{i+j}\left(V \times W \cup(M \times M-d(M)), S \times W \cup V \times T \cup(M \times M-d(M)) \stackrel{d !}{\rightarrow} H_{i+j-n}(V \cap W,(S \cap\right.$ $W) \cup(V \cap T))$,

onde d! é a função transfer de d. A composição de funções acima, multiplicada por $(-1)^{n(n-j)}$, é chamada interseção de pares e denotado por $\bullet$

\section{Propriedades:}

1. (comutatividade) Dados $\xi \in H_{n-i}(V, S)$ e $\eta \in H_{i}(W, T)$ então $\xi \bullet \eta=(-1)^{(n-|\xi|)(n-|\eta|)} \eta \bullet$ $\xi$.

2. (associatidade)

$$
(\xi \bullet \eta) \bullet \epsilon=\xi \bullet(\eta \bullet \epsilon)
$$

3. (unidade) Dados $\xi \in H_{n-i}(V, S)$ e $\omega \in H(M, M-K)$ é uma classe fundamental em volta de algum compacto $K$ tal que $(V-S) \subset K \in M$. Então $\omega \bullet \xi=\xi=\xi \bullet \omega$

4. (estabilidade) O seguinte diagrama é comutativo,

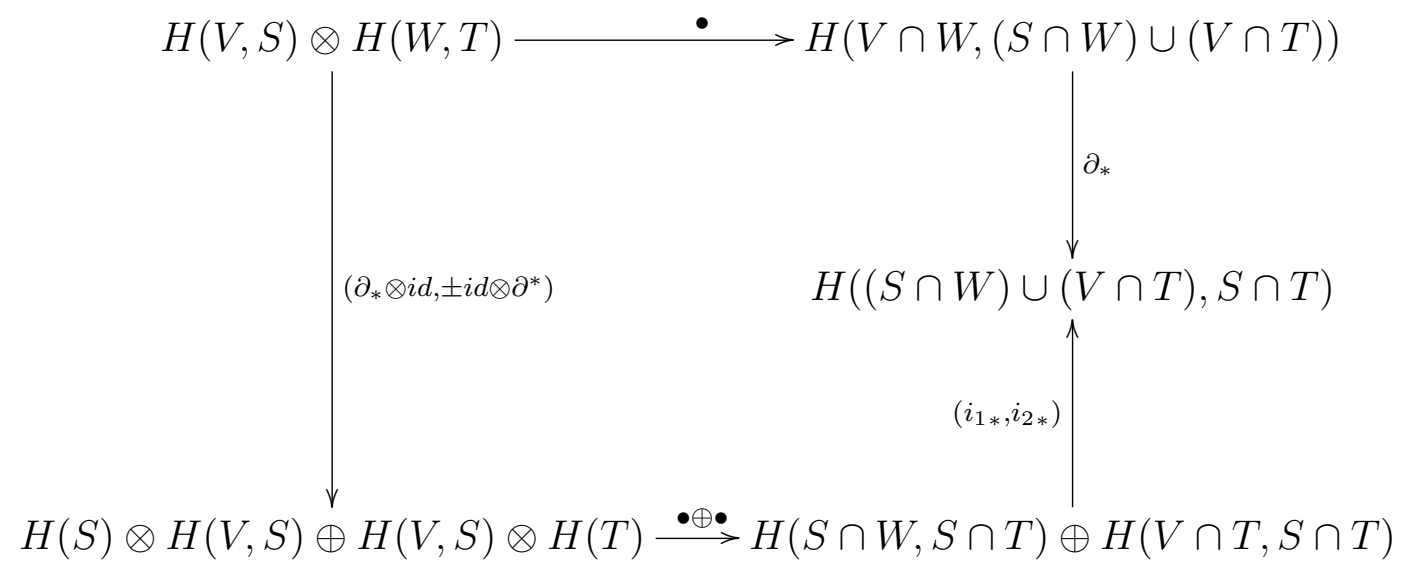


onde $i_{1 *}$ e $i_{2 *}$ são induzidas das inclusões. Isto é,

$\partial(\xi \bullet \eta)=i_{1 *}\left[\left(\partial_{*} \xi\right) \bullet \eta\right]+(-1)^{n-|\xi|} i_{2 *}\left[\xi \bullet\left(\partial_{*} \eta\right)\right]$

5. (multiplicidade) Sejam $M^{n}, M^{\prime n^{\prime}}$ variedades orientáveis, $\xi, \eta$ e $\xi^{\prime}, \eta^{\prime}$ classes de homologia de pares de abertos de $M$ e $M^{\prime}$, respectivamente. Então

$$
\left(\xi \times \xi^{\prime}\right) \bullet\left(\eta \times \eta^{\prime}\right)=(-1)^{\left(n^{\prime}-\left|\xi^{\prime}\right|\right)(n-|\eta|)}(\xi \bullet \eta) \times\left(\xi^{\prime} \bullet \eta^{\prime}\right)
$$

Proposição 1.6.3. Sejam $N_{1}, N_{2}$ subvariedades orientáveis compactas de uma variedade orientada $M^{m}$ de respectivas dimensões $n_{1}, n_{2}$ tal que $N=N_{1} \cap N_{2}$ é uma variedade conexa, compacta e orientável de dimensão $n=n_{1}+n_{2}-m$.

Sejam as classes fundamentais

$$
o_{N}^{N_{l}} \in H_{n_{l}}\left(N_{l}, N_{l}-N\right), l=1,2 .
$$

Se $N_{1}, N_{2}$ são transversais em um ponto $P \in N$. Então $o_{N}^{N_{1}} \bullet o_{N}^{N_{2}}= \pm o_{N}^{N}\left(o_{N}^{N} \in\right.$ $\left.H_{n}(N, \mathbb{Z})\right)$.

Teorema 1.6.1. Sejam uma variedade orientável $M$ e $N \subset M$ uma subvariedade compacta orientável. Se existir uma outra subvariedade orientável $N^{\prime}=\bar{N}^{\prime}$ de $M$ tal que $N^{\prime}$ intersecta transversalmente $N$ e $N \cap N^{\prime}$ não é nulo homólogo em $N^{\prime}$, então $N$ não é nulo homólogo em $M$, isto é, $i_{*}\left(o_{N}\right) \neq 0$,onde $i: N \rightarrow M$ é a inclusão natural e $o_{N} \in H(N)$ é uma classe fundamental.

Outros resultados podem ser encontrados em [3]. 


\section{Capítulo}

2

\section{Mergulhos de produto de esferas em}

\section{esferas}

\subsection{Mergulhos PL do Toro em $S^{3}$}

Neste capítulo vamos estudar os mergulhos de $S^{p} \times S^{p}$ em $S^{p+q+1}$. Inicialmente, $S^{1} \times S^{1}$ em $S^{3}$. Quando tem-se um mergulho PL $f$ de $T^{2}$ em $S^{3}$, f divide $S^{3}$ em dois lados, o parte de "dentro" de $f\left(T^{2}\right)$ e a parte de "fora" de $f\left(T^{2}\right)$ (que contém o ponto $\infty$ ), os quais seus bordos são $f\left(T^{2}\right)$. A questão é, será que se podemos mergulhar o toro de uma forma muito complicada em $S^{3}$ ? a resposta é dada pelo teorema principal desta seção que diz que somente podemos ter mergulhos PL tal que um dos lados, unido com $f\left(T^{2}\right)$, seja homeomorfo a um toro solido, isto é $S^{1} \times D^{2}$.

Definição 2.1.1. Seja $X$ espaço topológico e $Y \subset X$ um subconjunto. $Y$ é dito ser bicolarizada $($ em $X)$ se existe um mergulho $b: Y \times[-1,1] \rightarrow X$ tal que $b(y, 0)=y$, $\forall y \in Y$. A função b, ou melhor sua imagem, é o bicolar de $Y$ em $X$.

Os próximos teoremas são usados na prova do teorema 2.1.5. As demonstrações destes teoremas podem ser encontradas em [25]. Para os teoremas 2.1.1 e 2.1.2 ver apêndice B.

Teorema 2.1.1 (Teorema do loop). Se $M$ é uma 3-variedade com bordo e $i_{*}: \pi_{1}(\partial M) \rightarrow$ $\pi_{1}(M)$, induzida da inclusão $i: \partial M \rightarrow M$, tem núcleo não trivial, então existe um 
mergulho $f$ do disco $D=\left\{x \in \mathbb{R}^{2} ;|x| \leq 1\right\}$ em $M$ tal que $f(\partial D) \subset \partial M$ e $f(\partial D)$ é um elemento não nulo de $\pi_{1}(\partial M)$.

Dada uma função $f: D \rightarrow M$, o conjunto de pontos singulares de $f$ será denotado por $S(f)=\{x \in D ; \exists y \in D ; y \neq x$ e $f(x)=f(y)\}$.

Teorema 2.1.2 (Lema de Dehn). Suponha que $M$ é uma 3-variedade topológica e $f$ : $D \rightarrow M$ é uma função contínua $P L$ tal que $\overline{S(f)} \cap \partial D=\emptyset$. Então existe um mergulho $g: D \rightarrow M$ tal que $g(\partial D)=f(\partial D)$.(Onde $\overline{S(f)}$ significa o fecho de $S(f))$.

Corolário 2.1.2.1. Seja $M$ 3-variedade com $\partial M \neq \emptyset$. Suponha $J \subset \partial M$ é uma curva fechada e que $J$ é homotopicamente nula em $M$. Então existe um mergulho $P L f: D \rightarrow M$ tal que $\partial(f(D))=J \subset \partial M e(f \stackrel{\circ}{D})) \subset \stackrel{\circ}{M}$.

Teorema 2.1.3 (Teorema Generalizado de Schöenflies). Suponha que $K^{n-1}$ é uma $(n-1)$ esfera bicolarizada em $\mathbb{R}^{n}$. Então o fecho do complementar da parte limitada por $K^{n-1}$ é homeomorfo a n-bola $B^{n}$.

Uma prova deste teorema foi feita por Morton Brown em 1960 em [5].

Proposição 2.1.1. Quando costuramos um $D^{2} \times[0,1]$ a uma 3-bola, de forma que quando costurar $D^{2} \times 0$ e $D^{2} \times 1$ sejam disjunto nos bordos, o resultado, se orientável, é homeomorfo ao toro sólido $D^{2} \times S^{1}$.

Teorema 2.1.4. Dado $f$ um mergulho $P L$ de $S^{1} \times S^{1}=T^{2}$ em $S^{3}$. Então $X=S^{3}-f\left(T^{2}\right)$ têm duas componentes conexas por caminhos.

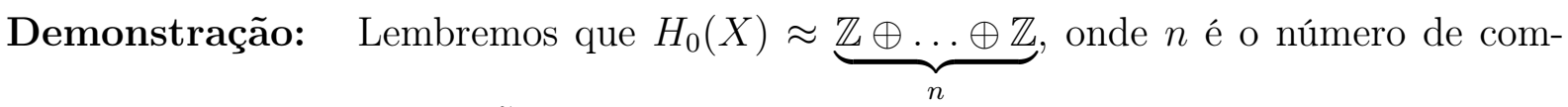
ponentes conexas de $X$ e $\tilde{H}_{0}(X) \oplus \mathbb{Z} \approx H_{0}(X)$. Agora pela dualidade de Alexander temos

$$
\tilde{H}^{k}\left(f\left(T^{2}\right)\right) \approx \tilde{H}_{3-k-1}(X)=\tilde{H}_{2-k}(X) .
$$

Assim temos

$$
\tilde{H}_{0}(X) \approx \tilde{H}^{2}\left(f\left(T^{2}\right)\right) \approx \tilde{H}^{2}\left(S^{1} \times S^{1}\right) \approx H^{2}\left(S^{1} \times S^{1}\right) \approx \mathbb{Z}
$$


Logo

$$
\tilde{H}_{0}(X) \approx \mathbb{Z} \Rightarrow H_{0}(X) \approx \mathbb{Z} \oplus \mathbb{Z}
$$

Portanto $X$ têm duas componentes conexas por caminhos.

Denotaremos estas componentes de $X$ por $C_{1}^{\prime}$ e $C_{2}^{\prime}$ e seus fechos de $C_{1}$ e $C_{2}$.

Teorema 2.1.5 (Teorema do toro de Alexander). Dado $f$ um mergulho $P L$ de $S^{1} \times S^{1}=$ $T^{2}$ em $S^{3}$. Consideremos os fechos das componentes conexas $C_{1}$ e $C_{2}$ tal que $C_{1} \cup C_{2}=S^{3}$ e $C_{1} \cap C_{2}=f\left(T^{2}\right)$. Então pelo menos uma destes fechos é homeomorfo ao toro sólido $D^{2} \times S^{1}$.

Demonstração: Sejam $C_{1}$ e $C_{2}$ os fechos das duas componentes, então $C_{1} \cup C_{2}=S^{3}$ e $T=C_{1} \cap C_{2}=f\left(T^{2}\right)$.

Consideremos os homomorfismos induzidos das inclusões $i_{1 *}: \pi_{1}(T) \rightarrow \pi_{1}\left(C_{1}\right), i_{2 *}$ : $\pi_{1}(T) \rightarrow \pi_{1}\left(C_{2}\right), j_{1_{*}}: \pi_{1}\left(C_{1}\right) \rightarrow \pi_{1}\left(S^{3}\right), j_{2 *}: \pi_{1}\left(C_{2}\right) \rightarrow \pi_{1}\left(S^{3}\right)$ e o seguinte diagrama

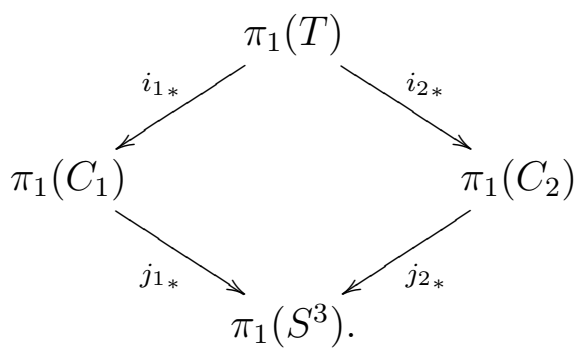

Se $i_{1 *}$ e $i_{2 *}$ forem injetivas, então pelo Corolário 1.1.1.1, $j_{1 *}$ e $j_{2 *}$ também serão injetivas e assim $\pi_{1}\left(T^{2}\right) \simeq \mathbb{Z} \oplus \mathbb{Z}$ seria um subgrupo de $\pi_{1}\left(S^{3}\right) \simeq 0$, o que é um absurdo.

Portanto, sem perda de generalidade, podemos assumir que $i_{1 *}$ não seja injetiva. Observemos que $C_{1}$ e $C_{2}$ são 3 -variedades com bordo igual a $T$. Logo, pelo Teorema 2.1.1, como $i_{1 *}$ não é injetiva, existe um mergulho $f_{1}: D^{2} \rightarrow C_{1}$ tal que $J=f_{1}\left(\partial D^{2}\right) \subset T$ e $J$ representa um elemento não trivial em $\pi_{1}(T)$.

Como $J$ está no bordo de um 2-disco em $C_{1}$ segue $J$ é homotopicamente nula em $\pi_{1}\left(C_{1}\right)$. Pelo Corolário 2.1.2.1 existe um mergulho $g_{1}: D^{2} \rightarrow C_{1}$ tal que $\partial D=J \subset \partial C_{1}$ e 
$\stackrel{\circ}{D} \subset \stackrel{\circ}{C}_{1}$, onde $D=g_{1}\left(D^{2}\right)$ e $g_{1}$ é PL.

Seja $b: D \times[-1,1] \rightarrow C_{1}$ um mergulho PL tal que $b(y, 0)=y, \forall y \in D$, cuja imagem é denotada por $N$, isto é, $N$ é uma vizinhança bicolar de $D$ em $C_{1}$. Então $N \cap T=b(J \times[-1,1])$ é uma vizinhança anular de $J$ em $T$. Desde que $J$ é não trivial em $\pi_{1}(T)$, temos $T-N$ uma vizinhança anular. Logo $\partial\left(C_{1}-N\right)$ é a união de dois 2-discos PL que estão no $\partial N$ e o conjunto $T-N$ que é uma vizinhança anular. Ver desenho abaixo.

Portanto $C_{1}-N$ é bordado por uma 2-esfera PL em $S^{3}$. Como $S^{3}$ pode ser vista como $\mathbb{R}^{3}$ compactificado no infinito e é limitada, pelo teorema $2.1 .3 C_{1}-N$ é PL homeomorfo a uma 3-bola $B^{3}$. Para completar a prova basta notar que $C_{1}$ é orientável e pela proposição 2.1.1 $C_{1}$ é homeomorfo a $D^{2} \times S^{1}$.

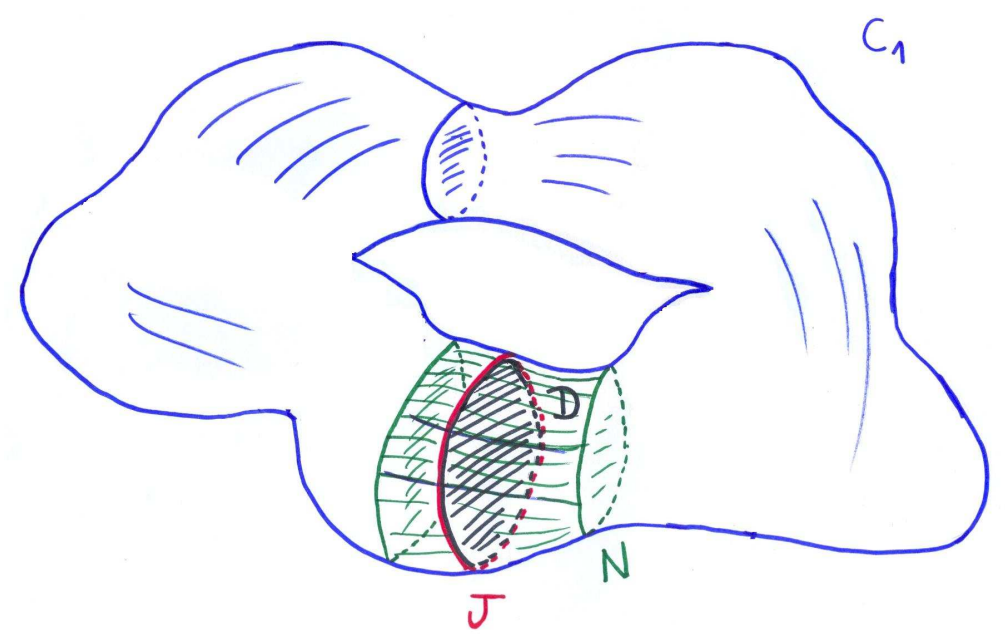

Usamos [25] como referência nesta seção, por ser um jeito mais simples de demonstrar o Teorema 2.1.5. Mas James Alexander, daí o nome teorema do toro de Alexander, demonstrou este teorema em [1].

\subsection{Mergulhos de $S^{p} \times S^{q}$ em $S^{p+q+1}$}

A questão abordada na seção anterior, sobre a caracterização do complementar de certos mergulhos, gerou algumas questões sobre generalizações. [13] e [9], começaram a aplicar a Teoria desenvolvida por Smale no famoso Teorema do h-cobordismo. Depois 
Laércio Ap. Lucas, Osamu Saeki e Oziride M. Neto, nos artigos [20], [18] e [19], trabalharam sobre estas caracterizações de mergulhos de produtos de esferas em esferas. Fizeram isto na categoria diferenciável.

Seja $f: S^{p} \times S^{q} \rightarrow S^{p+q+1}$ um mergulho suave. Mostraremos que uma das componentes conexas por caminhos de $S^{p+q+1}-f\left(S^{p} \times S^{q}\right)$, se $p \geq q \geq 1$ e $p+q \neq 3$, é difeomorfa a $S^{p} \times D^{q+1}$ e é homeomorfa a $S^{2} \times D^{2}$ se $p=2$ e $q=1$. Este resultado generaliza o teorema 2.1.5. Dividiremos em casos de $p$ e $q$ para demonstrar o teorema principal.

Proposição 2.2.1. Seja um mergulho suave $f: S^{p} \times S^{q} \rightarrow S^{p+q+1}$, onde $p \geq q \geq 1$. Então $S^{p+q+1}-f\left(S^{p} \times S^{q}\right)$ tem duas componentes conexas.

Demonstração: Seja $E=S^{p+q+1}-f\left(S^{p} \times S^{q}\right)$. Temos

$$
H_{0}(E) \approx \tilde{H}_{0}(E) \oplus \mathbb{Z} \approx \underbrace{\mathbb{Z} \oplus \ldots \oplus \mathbb{Z}}_{n+1}
$$

onde $n$ é o número de componentes conexas.

Pela dualidade de Alexander

$$
\tilde{H}_{0}(E) \approx \tilde{H}^{p+q+1-1}\left(f\left(S^{p} \times S^{q}\right)\right) \approx \tilde{H}^{p+q}\left(S^{p} \times S^{q}\right) \approx \mathbb{Z}
$$

Logo $H_{0}(E) \approx \mathbb{Z} \oplus \mathbb{Z}$. Portanto $E$ tem duas componentes conexas.

\subsubsection{Caso em que $p>q \geq 2$}

Denotemos as duas componentes conexas por caminhos de $E=S^{p+q+1}-f\left(S^{p} \times S^{q}\right)$ por $C_{1}^{\prime}$ e $C_{2}^{\prime}$ e os seus respectivos fechos em $S^{p+q+1}$ chamaremos de $C_{1}$ e $C_{2}$. Primeiramente consideremos o caso em que $p>q \geq 2$ e com alguns resultados caracterizaremos $C_{1}$ e $C_{2}$.

Teorema 2.2.1. Uma das componentes, $C_{1}$ ou $C_{2}$, tem a homologia de $S^{p}$ e a outra tem a homologia de $S^{q}$. 
Demonstração: Primeiramente observemos que do fato de $C_{1}$ é uma variedade compacta suave com bordo e pelo teorema 1.5.4 segue que $H_{*}\left(C_{1}^{\prime}\right) \approx H_{*}\left(C_{1}\right)$. Analogamente para $H_{*}\left(C_{2}^{\prime}\right)=H_{*}\left(C_{2}\right)$.

Agora, suponhamos que $\tilde{H}_{i}\left(C^{\prime}{ }_{1}\right) \approx \tilde{H}_{i}\left(C_{i}\right)=0 \forall i \in \mathbb{Z}$. Consideremos os conjuntos $\Sigma_{1}=f\left(S^{p} \times\left\{x_{1}\right\}\right)$ e $\Sigma_{2}=f\left(\left\{x_{2}\right\} \times S^{q}\right)$ os quais são subvariedades mergulhadas em $\partial C_{1}=f\left(S^{p} \times S^{q}\right)$ e se interceptam em um único ponto $x=\left(x_{2}, x_{1}\right)$.

Sabemos que $T_{x}\left(\Sigma_{1}\right)+T_{x}\left(\Sigma_{2}\right) \approx T_{x_{2}}\left(S^{p}\right) \times\{1\}+\{1\} \times T_{x_{1}}\left(S^{q}\right) \approx T_{x_{2}}\left(S^{p}\right)+T_{x_{1}}\left(S^{q}\right) \approx$ $T_{x}\left(S^{p} \times S^{q}\right)$.

Portanto $\Sigma_{1}$ e $\Sigma_{2}$ são transversais em $f\left(S^{p} \times S^{q}\right)$.

Como $H_{p}\left(C_{1}\right) \approx 0$ então existe um $(\mathrm{p}+1)$-ciclo $(D, \partial D)$ em $\left(C_{1}, \partial C_{1}\right)$ cujo bordo é homólogo a $\Sigma_{1}$ em $\partial C_{1}$, isto é, $[\partial D]=\left[\Sigma_{1}\right]$ em $\partial C_{1}$.

Logo $D \cap \Sigma_{2}=x$ e como $\Sigma_{1}$ é transversal a $\Sigma_{2}$ segue que $D$ e $\Sigma_{2}$ são transversais em $C_{1}$.

Além disso, temos:

(i) $C_{1}$ é uma variedade compacta orientável e $\Sigma_{2}$ é uma subvariedade orientável;

(ii) existe $D$, subvariedade orientável de $C_{1}$, tal que $D$ intersecta transversalmente $\Sigma_{2}$. Além disso, $\Sigma_{2} \cap D=\{x\}$ não é nulo homologa em $\Sigma_{2}$, isto é, dada a função

$$
\begin{gathered}
i_{*}: H_{0}(\{x\} ; Z) \approx \mathbb{Z} \rightarrow H_{0}\left(\Sigma_{2} ; \mathbb{Z}\right) \approx \mathbb{Z} \\
{[x] \longmapsto[x]}
\end{gathered}
$$

temos $i_{*}([x])=[x] \neq 0$

Pelo teorema 1.6.1, $i_{*}\left(\left[\Sigma_{2}\right]\right)=\left[\Sigma_{2}\right] \neq 0$, onde $i_{*}: H_{q}\left(\partial C_{1} ; \mathbb{Z}\right) \rightarrow H_{q}\left(C_{1} ; \mathbb{Z}\right)$ é a induzida da inclusão.

Portanto $H_{q}\left(C_{1}\right) \neq 0$ o que contradiz a hipótese. Além disso, provamos que quando $H_{p}\left(C_{1}\right)=0$ então $H_{q}\left(C_{1}\right) \neq 0$.

Logo $H_{i}\left(C_{1}\right) \neq 0$ para algum $i \in \mathbb{Z}$.

Fazendo análogo temos o mesmo resultado para $C_{2}$, isto é, quando $H_{q}\left(C_{2}\right)=0$ então $H_{p}\left(C_{2}\right) \neq 0$. 
Agora seja $E=S^{p+q+1}-f\left(S^{p} \times S^{q}\right)$, então pela dualidade de Alexander temos:

$$
\tilde{H}_{i}(E) \approx \tilde{H}^{p+q+1-i-1}\left(f\left(S^{p} \times S^{q}\right)\right) \approx \tilde{H}^{p+q-i}\left(S^{p} \times S^{q}\right) .
$$

Portanto tem-se dois casos:

Caso 1) $p \neq q$

$$
\tilde{H}_{i}(E) \approx \begin{cases}\mathbb{Z}, & \text { quando } i=q, i=p \text { e } i=0 \\ 0, & \text { caso contrário }\end{cases}
$$

Caso 2) $p=q$

$$
\tilde{H}_{i}(E) \approx \begin{cases}\mathbb{Z} \oplus \mathbb{Z} & , \quad \text { se } i=p=q \\ \mathbb{Z} & , \quad \text { se } i=0 \\ 0 & , \quad \text { caso contrário }\end{cases}
$$

Desde que $C_{1}$ e $C_{2}$ são componentes conexas de $E, \tilde{H}_{i}(E) \approx \tilde{H}_{i}\left(C_{1}\right) \oplus \tilde{H}_{i}\left(C_{2}\right)$.

No Caso 1) temos os seguintes:

- $\tilde{H}_{p}(E) \approx \mathbb{Z} \approx \tilde{H}_{p}\left(C_{1}\right) \oplus \tilde{H}_{p}\left(C_{2}\right)$ o que implica duas possibilidade, que são

(i) $\tilde{H}_{p}\left(C_{1}\right) \approx \mathbb{Z}$ e $\tilde{H}_{p}\left(C_{2}\right) \approx 0 \mathrm{ou}$

(ii) $\tilde{H}_{p}\left(C_{1}\right) \approx 0$ e $\tilde{H}_{p}\left(C_{2}\right) \approx \mathbb{Z}$

- $\tilde{H}_{q}(E) \approx \mathbb{Z} \approx \tilde{H}_{q}\left(C_{1}\right) \oplus \tilde{H}_{q}\left(C_{2}\right)$

- se $(i)$ então $\tilde{H}_{q}\left(C_{1}\right) \approx 0$ e $\tilde{H}_{q}\left(C_{2}\right) \approx \mathbb{Z}$

- se $(i i)$ então $\tilde{H}_{q}\left(C_{1}\right) \approx \mathbb{Z}$ e $\tilde{H}_{q}\left(C_{2}\right) \approx 0$

- $H_{0}(E) \approx \tilde{H}_{0}(E) \oplus \mathbb{Z} \approx \mathbb{Z} \oplus \mathbb{Z} \approx H_{0}\left(C_{1}\right) \oplus H_{0}\left(C_{2}\right) \Rightarrow H_{0}\left(C_{1}\right) \approx \mathbb{Z}$ e $H_{0}\left(C_{2}\right) \approx \mathbb{Z}$.

- $\tilde{H}_{i}\left(C_{1}\right) \approx \tilde{H}_{i}\left(C_{2}\right) \approx 0$, caso contrário.

No Caso 2) temos os seguintes:

- $\tilde{H}_{p}(E) \approx \mathbb{Z} \oplus \mathbb{Z} \approx \tilde{H}_{p}\left(C_{1}\right) \oplus \tilde{H}_{p}\left(C_{2}\right) \Rightarrow H_{p}\left(C_{1}\right) \approx \mathbb{Z}$ e $H_{p}\left(C_{2}\right) \approx \mathbb{Z}$. Igualmente para $H_{q}\left(C_{1}\right) \approx H_{q}\left(C_{2}\right) \approx \mathbb{Z}$, pois $p=q$. 
- $H_{0}(E) \approx \tilde{H}_{0}(E) \oplus \mathbb{Z} \approx \mathbb{Z} \oplus \mathbb{Z} \approx H_{0}\left(C_{1}\right) \oplus H_{0}\left(C_{2}\right) \Rightarrow H_{0}\left(C_{1}\right) \approx \mathbb{Z}$ e $H_{0}\left(C_{2}\right) \approx \mathbb{Z}$.

- $\tilde{H}_{i}\left(C_{1}\right) \approx \tilde{H}_{i}\left(C_{2}\right) \approx 0$, caso contrário.

Portanto em ambos os casos ou $C_{1}$ tem a homologia de $S^{p}$ e $C_{2}$ tem a homologia de $S^{q}$ ou, ao contrário, $C_{1}$ tem a homologia de $S^{q}$ e $C_{2}$ tem a homologia de $S^{p}$.

Daqui por diante assumiremos que $C_{1}$ tem a homologia de $S^{p}$ e que $C_{2}$ tem a homologia de $S^{q}$.

Consideremos o conjunto $\Sigma_{1}=f\left(S^{p} \times\{*\}\right) \subset \partial C_{1}$. Através de um campo vetorial normal $s$, apontando para $\operatorname{Int}\left(C_{1}\right)$, de $f\left(S^{p} \times S^{q}\right)$ em $S^{p+q+1}$, "empurramos" $\Sigma_{1}$ para o interior de $C_{1}$, como sendo a imagem de $\Sigma_{1}$ por $s$, e denotemos $\Sigma_{1}^{\prime}$ para o $\Sigma_{1}$ empurrado para o interior. Segue do fato de que quando restringimos $s$ à sua imagem, $s$ será um homeomorfismo e, portanto, $\Sigma_{1}^{\prime} \cong \Sigma_{1} \operatorname{com} \Sigma_{1}^{\prime} \in \operatorname{Int}\left(C_{1}\right)$. Como $\Sigma_{1}^{\prime}$ é homeomorfa a $\Sigma_{1}$ segue que $\Sigma_{1}^{\prime}$ é compacta e portanto existe uma vizinhança tubular de $\Sigma_{1}^{\prime}$, denotada por $G$, suficientemente pequena de forma que $G \subset \operatorname{Int}\left(C_{1}\right)$. Veja desenho abaixo.

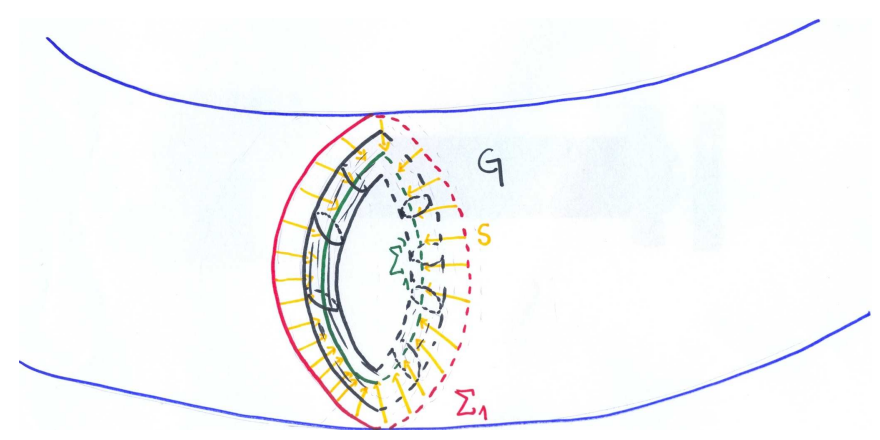

Lema 2.2.1. A variedade $G$ é sempre difeomorfa a $S^{p} \times D^{q+1}$

Demonstração: Como o fibrado normal de $S^{p} \times\{*\}$ em $S^{p+q+1}$ é trivial e $f$ é um mergulho suave, segue que o fibrado normal de $\Sigma_{1}$ em $S^{p+q+1}$ é trivial também. Portanto, como $\Sigma_{1}^{\prime} \cong \Sigma_{1}, G$ é difeomorfo a $\left(S^{p} \times\{*\}\right) \times D^{q+1}$ que por sua vez é difeomorfo a $S^{p} \times D^{q+1}$.

Denotaremos $i: \partial C_{1} \rightarrow C_{1}$ a função inclusão. 
Lema 2.2.2. A classe $i_{*}\left(\left[\Sigma_{1}\right]\right)$ é o gerador de $H_{p}\left(C_{1}\right)$ quando $p>q$, onde $\left.\left[\Sigma_{1}\right] \in H_{p}\left(\partial C_{1}\right)\right]$ é a classe de homologia representado por $\Sigma_{1}$.

Demonstração: Como $H_{q}\left(C_{1}\right) \approx 0$ então existe um q +1 -ciclo $(D, \partial D) \in\left(C_{1} \partial C_{1}\right)$ cujo bordo é homólogo a $\Sigma_{2}=f\left(\{*\} \times S^{q}\right)$ em $C_{1}$, isto é $\left[\Sigma_{2}\right]=[\partial D]$. Fazendo da mesma forma como foi feito na demonstração da proposição 2.2.1, segue que o número interseção entre $[(D, \partial D)]$ e $\left[\Sigma_{1}\right] \in H_{p}\left(C_{1}\right)$ é igual a \pm 1 e, portanto, $i_{*}\left(\left[\Sigma_{1}\right]\right)$ é não nula. Como $C_{1}$ é uma variedade compacta e $\left(C_{1}, \partial C_{1}\right)$ são orientáveis segue da dualidade de Poincaré-Lefschetz que $H_{p}\left(C_{1}, \partial C_{1}\right) \approx H^{q+1}\left(C_{1}\right) \approx\{0\}$. Consideremos a sequência abaixo:

$$
0 \rightarrow \operatorname{Ker}\left(i_{*}\right) \rightarrow H_{p}\left(\partial C_{1}\right) \stackrel{i_{*}}{\rightarrow} H_{p}\left(C_{1}\right) \rightarrow H_{p}\left(C_{1}, \partial C_{1}\right) \approx\{0\}
$$

que é exata e sendo $H_{p}\left(C_{1}\right)$ livre, então a sequência cinde, isto é, $\mathbb{Z} \approx H_{p}\left(\partial C_{1}\right) \approx \operatorname{Ker}\left(i_{*}\right) \oplus$ $\beta$, onde $\beta$ é subgrupo de $H_{p}\left(C_{1}\right)$. Portanto temos dois casos que podem ocorrer (i) $\operatorname{Ker}\left(i_{*}\right) \approx\{0\}$ e $\beta \approx \mathbb{Z}$ ou (ii) $\operatorname{Ker}\left(i_{*}\right) \approx \mathbb{Z}$ e $\beta \approx\{0\}$. Agora, (ii) não pode ocorrer pois $i_{*}\left(\left[\Sigma_{1}\right]\right) \neq 0$. Logo temos (i), mas então $i_{*}$, que já é sobrejetiva pela sequência, é injetiva e portanto um isomorfismo. Dessa forma $i_{*}\left(\left[\Sigma_{1}\right]\right)$ é gerador de $H_{p}\left(C_{1}\right)$.

Consideremos o conjunto $V=\overline{C_{1}-G}$

Lema 2.2.3. Quando $p>q \geq 2$. Então $\partial C_{1}$, $\partial G$ e $V$ são simplesmente conexos. Além disso $V$ é um h-cobordismo entre $\partial G$ e $\partial C_{1}=f\left(S^{p} \times S^{q}\right)$.

Demonstração: Como $p>q \geq 2$ e $\partial C_{1}$ e $\partial G$ são difeomorfos respectivamente a $S^{p} \times S^{q}$ e $S^{p} \times S^{q}$, segue que

$$
\pi_{1}\left(\partial C_{1}\right) \approx \pi_{1}\left(S^{p} \times S^{q}\right) \approx \pi_{1}\left(S^{p}\right) \times \pi_{1}\left(S^{q}\right) \approx\{1\}
$$

e

$$
\pi_{1}(\partial G) \approx \pi_{1}\left(S^{p} \times S^{q}\right) \approx \pi_{1}\left(S^{p}\right) \times \pi_{1}\left(S^{q}\right) \approx\{1\}
$$

Agora desde que a dimensão de $\Sigma_{1}^{\prime} \in \operatorname{Int}\left(C_{1}\right)$ é igual $p$, então a codimensão é igual a $(p+q+1)-p=q+1 \geq 3$. Assim temos $\pi_{1}\left(C_{1}-\Sigma_{1}^{\prime}\right) \approx \pi_{1}\left(C_{1}\right)$. Além disso temos $D^{q+1}$ 
é contrátil e portanto $\Sigma_{1}^{\prime}$ é um retrato de $G$ e segue que $\pi_{1}\left(C_{1}-\Sigma_{1}^{\prime}\right) \approx \pi_{1}(V)$.

Temos ainda que $C_{1} \cap C_{2}=f\left(S^{p} \times S^{q}\right)$ e $C_{1} \cup C_{2}=S^{p+q+1}$ são simplesmente conexos, pelo teorema 1.1.1 temos $C_{1}$ e $C_{2}$ também simplesmente conexos.

Consideremos $G \subset C_{1}$ e $U=C_{1}-V$ então $U=\operatorname{Int}(G)$ e $\bar{U}=G \subset G$. Logo por excisão

$$
H_{*}\left(C_{1}-U, G-U\right)=H_{*}(V, \partial G) \approx H_{*}\left(C_{1}, G\right)
$$

Sabemos ainda que $H_{*}\left(C_{1}\right) \approx H_{*}\left(\Sigma_{1}\right) \approx H_{*}(G) \approx H_{*}\left(S^{p}\right)$ e pela sequência do par $\left(C_{1}, G\right)$

$$
\ldots H_{r}(G) \stackrel{\approx}{\rightarrow} H_{r}\left(C_{1}\right) \rightarrow H_{r}\left(C_{1}, G\right) \rightarrow H_{r-1}(G) \stackrel{\approx}{\rightarrow} H_{r-1}\left(C_{1}\right) \rightarrow \ldots
$$

Portanto $H_{*}\left(C_{1}, G\right) \approx\{0\}$.

Com isso obtemos as seguintes propriedades:

(i) $\partial G, \partial C_{1}$ e $V$ são simplesmente conexos.

(ii) $H_{*}(V, \partial G) \approx\{0\}$

(iii) $\operatorname{dim} V=p+q+1 \geq 6$.

Então pelo teorema 1.5.6 $V$ é uma h-cobordismo entre $\partial G$ e $\partial C_{1}$.

Além disso, pelo teorema 1.5.6, temos $V$ difeomorfo a $\partial G \times[0,1]$ e $C_{1}=G \cup V \cong$ $G \cup(\partial G \times[0,1]) \cong G \cup \partial G \cong G$. Segue do lema 2.2.1 que $G$ é difeomorfo a $S^{p} \times D^{q+1}$.

Usando-se os mesmos argumentos da prova de que $C_{1} \cong S^{p} \times D^{q+1}$, mostra-se que $C_{2} \cong D^{p+1} \times S^{q}$.

Com isso, obtém-se o seguinte resultado:

Teorema 2.2.2. Seja $M$ uma $(p+q+1)$-variedade compacta e simplesmente conexa, com $p>q \geq 2$, satisfazendo as seguintes prorpiedades:

1. $\partial M$ é difeomorfo a $S^{p} \times S^{q}$,

2. $H_{*}(M) \approx H_{*}\left(S^{p}\right)$ ou $H_{*}(M) \approx H_{*}\left(S^{q}\right)$.

Então $M$ é difeomorfo a $S^{p} \times D^{q+1}$, se $H_{*}(M) \approx H_{*}\left(S^{p}\right)$, e se $H_{*}(M) \approx H_{*}\left(S^{q}\right) M$ é difeomorfo a $D^{p+1} \times S^{q}$ 


\subsubsection{Caso em que $p=q \geq 2$}

No caso $p=q \geq 2$, pelo Teorema 2.2.1, $C_{1}$ e $C_{2}$ têm a homologia de $S^{p}$. Além disso, como $p=q \geq 2$ tem-se que $\pi_{1}\left(S^{2 p+1}\right) \approx \pi_{1}\left(S^{p} \times S^{p}\right) \approx\{1\}$. Portanto pelo teorema 1.1.1, segue que $\pi_{1}\left(C_{1}\right) \approx \pi_{1}\left(C_{2}\right) \approx\{1\}$. Primeiramente vamos provar alguns resultados que serão no caso em que $p=q=2$ e no caso em que $p=q>2$.

Para os próximos resultados, considere uma $M(2 \mathrm{p}+1)$-variedade compacta e simplesmente conexa $(p \geq 2)$ satisfazendo:

(1) $\partial M \cong S^{p} \times S^{p}$

(2) $H_{*}(M) \approx H_{*}\left(S^{p}\right)$

Nestas condições, tem-se o seguinte resultado.

Teorema 2.2.3. Existe uma classe de homologia primitiva $\gamma \in H_{p}(\partial M)$ tal que $i_{*}(\gamma)=0$, onde $i: \partial M \rightarrow M$ é a inclusão.

Demonstração: Considere a seguinte sequência exata de homologia

$$
\ldots \rightarrow H_{p}(\partial M) \stackrel{i_{*}}{\rightarrow} H_{p}(M) \rightarrow H_{p}(M, \partial M) \rightarrow H_{p-1}(\partial M) \rightarrow \ldots
$$

Como $M$ é compacta e $(M, \partial M)$ é orientável segue, pela dualidade de Poincaré-Lefschetz, que $H_{p}(M, \partial M) \approx H^{(2 p+1)-p}(M)=H^{p+1}(M) \approx 0$, pois $M$ tem a homologia de $S^{p}$.

Considere a sequência exata

$$
0 \rightarrow \operatorname{Ker}\left(i_{*}\right) \rightarrow H_{p}(\partial M) \stackrel{i_{*}}{\rightarrow} H_{p}(M) \rightarrow 0
$$

Desde que $H_{p}(M) \approx \mathbb{Z}$ é livre segue que a sequência exata acima cinde, isto é, $\operatorname{Ker}\left(i_{*}\right) \oplus$ $\beta \approx H_{p}(\partial M) \approx \mathbb{Z} \oplus \mathbb{Z}$, onde $\beta$ é subgrupo de $H_{p}(M)$. Além disso, $\beta \neq 0$, pois caso contrário, $i_{*}$ não seria sobrejetiva. Portanto temos $\operatorname{Ker}\left(i_{*}\right) \approx \mathbb{Z}$.

Logo existe um elemento $\gamma \neq 0$ tal que $i_{*}(\gamma)=0$ e $\gamma$ é primitivo.

Lema 2.2.4. Quando p é par, o número interseção $\gamma \bullet \gamma$ é igual a zero. 
Demonstração: Usando a dualidade de Poincaré temos $\gamma \bullet \gamma=<\gamma^{*}, \gamma>$, onde $\gamma^{*} \in$ $H^{2 p-p}(\partial M)$ é o dual de Poincaré de $\gamma$.

Consideremos o seguinte diagrama

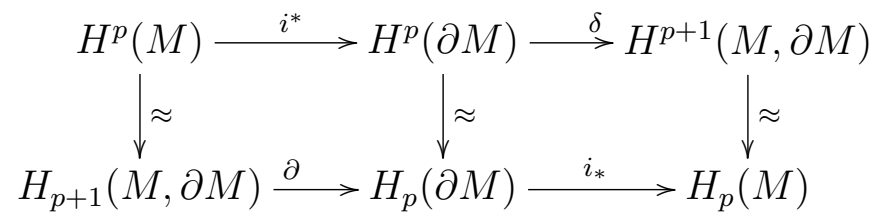

o qual é exato nas linhas horizontais e os isomorfismo verticais são dados pela dualidade de Poincaré-Lefschetz.

Desde que $i_{*}(\gamma)=0$ e do fato do diagrama comutar, temos $\delta\left(\gamma^{*}\right)=0$. Logo existe $\beta \in H^{p}(M)$ tal que $i^{*}(\beta)=\gamma^{*}$.

Dessa forma,

$\gamma \bullet \gamma=<\gamma^{*}, \gamma>=<i^{*}(\beta), \gamma>=<\beta, i_{*}(\gamma)>=<\beta, 0>=0$

Teorema 2.2.4. Existe uma classe de homologia $\delta \in H_{p}(\partial M)$ tal que $\gamma \bullet \delta=1$. Mais ainda, quando p é par, podemos escolher $\delta$ tal que $\delta \bullet \delta=0$.

Demonstração: Desde que $\gamma$ é uma classe primitiva e o rank de $H_{p}(\partial M)$ é dois, existe uma classe de homologia $\delta^{\prime} \in H_{p}(\partial M)$ tal que $\gamma \bullet \delta^{\prime}=1$ ou $\gamma \bullet \delta^{\prime}=-1$. Se necessário, mudamos a orientação de $\delta^{\prime}$ para termos $\gamma \bullet \delta^{\prime}=1$. Quando $p$ é par a forma interseção de $\partial M$ é do tipo par. $\operatorname{Logo} \delta^{\prime} \bullet \delta^{\prime}=2 m$ para algum $m \in \mathbb{Z}$. Tomemos $\delta=\delta^{\prime}-m \gamma$, assim temos,

$$
\begin{aligned}
\gamma \bullet \delta & =\gamma \bullet\left(\delta^{\prime}-m \gamma\right) \\
& =\gamma \bullet \delta^{\prime}-m(\gamma \bullet \gamma) \\
& =1-0 \\
& =1 .
\end{aligned}
$$

Além disso, temos

$$
\begin{aligned}
\delta \bullet \delta & =\left(\delta^{\prime}-m \gamma\right) \bullet\left(\delta^{\prime}-m \gamma\right) \\
& =\delta^{\prime} \bullet \delta^{\prime}-2 m\left(\delta^{\prime} \bullet \gamma\right)+m^{2}(\gamma \bullet \gamma) \\
& =2 m-2 m \\
& =0 .
\end{aligned}
$$


Consideremos $p$ par. Como $\partial M$ é difeomorfo a $S^{p} \times S^{p}$, então daqui por diante identificaremos $\partial M \operatorname{com} S^{p} \times S^{p}$. Seja $\xi_{1}=\left[S^{p} \times\{*\}\right]$ e $\xi_{2}=\left[\{*\} \times S^{p}\right]$, os quais geram $H_{p}(\partial M)=H_{p}\left(S^{p} \times S^{p}\right)$.

Escolhendo orientações convenientes para $S^{p} \times\{*\}$ e $\{*\} \times S^{p}$ temos $\xi_{1} \bullet \xi_{2}=1$ e $\xi_{i} \bullet \xi_{i}=0(i=1,2)$.

Como $\delta \bullet \delta=\gamma \bullet \gamma=0$ e $\delta \bullet \gamma=1$ segue que $\delta$ é gerador de $H_{p}(\partial M)$ e $\gamma \neq m \delta$, já que caso contrário $\gamma \bullet \delta=0$. Desde que rank de $H_{p}(\partial M)$ é $2,\{\delta, \gamma\}$ geram $H_{p}(\partial M)$.

Portanto o endomorfismo $\eta: H_{p}\left(S^{p} \times S^{p}\right) \rightarrow H_{p}\left(S^{p} \times S^{p}\right)$ definido por $\eta\left(\xi_{1}\right)=\delta$ e $\eta\left(\xi_{2}\right)=\gamma$ é um automorfismo de $H_{p}\left(S^{p} \times S^{p}\right)$.

Proposição 2.2.2. Desde que p é par então todo automorfismo de $H_{p}\left(S^{p} \times S^{p}\right.$, ) é realizado por um difeomorfismo que preserva a orientação.

Demonstração: Sejam $\eta: H_{p}\left(S^{p} \times S^{p}\right) \rightarrow H_{p}\left(S^{p} \times S^{p}\right)$ um automorfismo e $A=$ $\left(\begin{array}{ll}a & b \\ c & d\end{array}\right)$, onde $a, b, c$ e $d \in \mathbb{Z}$, a matriz de $\eta$ com respeito a base $\left\{\xi_{1}, \xi_{2}\right\}$. Temos ${ }^{t} A Q A=Q$, onde $Q$ é a matriz interseção de $S^{p} \times S^{p}$ com respeito a base $\left\{\xi_{1}, \xi_{2}\right\}$, isto é,

$$
Q=\left(\begin{array}{cc}
0 & 1 \\
1 & 0
\end{array}\right)=\left(\begin{array}{cc}
\xi_{1} \bullet \xi_{1} & \xi_{1} \bullet \xi_{2} \\
\xi_{2} \bullet \xi_{1} & \xi_{2} \bullet \xi_{2}
\end{array}\right) \mathrm{e}^{t} A=\left(\begin{array}{cc}
a & -c \\
-b & d
\end{array}\right)
$$

Temos o seguinte,

$$
\left(\begin{array}{ll}
a & b \\
c & d
\end{array}\right)\left(\begin{array}{l}
\xi_{1} \\
\xi_{2}
\end{array}\right)=\left(\begin{array}{c}
a \xi_{1}+b \xi_{2} \\
c \xi_{1}+d \xi_{2}
\end{array}\right)
$$

Como $A$ preserva número interseção segue que $\left(a \xi_{1}+b \xi_{2}\right) \bullet\left(a \xi_{1}+b \xi_{2}\right)=0,\left(a \xi_{1}+b \xi_{2}\right) \bullet$ $\left(c \xi_{1}+d \xi_{2}\right)=\left(c \xi_{1}+d \xi_{2}\right) \bullet\left(a \xi_{1}+b \xi_{2}\right)=1$ e $\left(c \xi_{1}+d \xi_{2}\right) \bullet\left(c \xi_{1}+d \xi_{2}\right)=0$. Logo obtém-se as seguintes equações

1) $2 a b=0$,

2) $a d+b c=1 \mathrm{e}$ 
3) $2 c d=0$.

Além disso, como $\eta$ é um automorfismo $\operatorname{det} A= \pm 1$, isto é, 4) $a d-b c= \pm 1$.

Portanto 1) implica que $a=0$ ou $b=0$. Primeiro suponhamos que $a=0$. De 2) temos $b c=1 \Rightarrow b=1$ e $c=1$ ou $b=-1$ e $c=-1$ e com isso de 3) $d=0$. Assim tem-se duas matrizes

$$
A=\left(\begin{array}{ll}
0 & 1 \\
1 & 0
\end{array}\right) \text { ou } A=\left(\begin{array}{cc}
0 & -1 \\
-1 & 0
\end{array}\right) \text {. }
$$

Suponha $b=0$, de 2) $a d=1 \Rightarrow a=1$ e $d=1$ ou $a=-1$ e $d=-1$ e de 3) $c=0$. Logo temos as outras duas possibilidades de $A$, que são

$$
A=\left(\begin{array}{ll}
1 & 0 \\
0 & 1
\end{array}\right) \text { ou } A=\left(\begin{array}{cc}
-1 & 0 \\
0 & -1
\end{array}\right)
$$

Para as quatro possibilidades da matriz $A$ temos os quatros respectivos difeomorfismos $h_{i}: S^{p} \times S^{p} \rightarrow S^{p} \times S^{p}, i=1,2,3,4$ dados por $h_{1}(x, y)=(y, x), h_{2}(x, y)=(-y,-x)$, $h_{3}=I d_{S^{p} \times S^{p}}$ e $h_{4}=-I d_{S^{p} \times S^{p}}$ os quais as realizam e além disso estes difeomorfismos preservam orientações.

Caso em que $p=q=2$

Apresentaremos alguns resultados que preparam o casa $p=q=2$. Nesta condição temos uma (5)-variedade $M$ compacta e simplesmente conexa satisfazendo:

(1) $\partial M \cong S^{2} \times S^{2}$

(2) $H_{*}(M) \approx H_{*}\left(S^{2}\right)$.

Sejam $\eta: H_{2}\left(S^{2} \times S^{2}\right) \rightarrow H_{2}\left(S^{2} \times S^{2}\right)$ um automorfismo e $\psi: S^{2} \times S^{2} \rightarrow S^{2} \times S^{2}$ um difeomorfismo que preserva orientação tal que $\psi_{*}=\eta$, o qual existe pela proposição 2.2.2.

Portanto podemos considerar $X=M \bigcup_{\psi} S^{2} \times D^{3}$, onde $\bigcup_{\psi}$ significa colagem pelos bordos, $\partial M \approx S^{2} \times S^{2}$ e $\partial\left(S^{2} \times D^{3}\right)=S^{2} \times S^{2}$, através de $\psi$, isto é, identificamos $\partial M$ e 
$S^{2} \times S^{2}$ pelo difeomorfismo $\psi$. Como na figura abaixo.

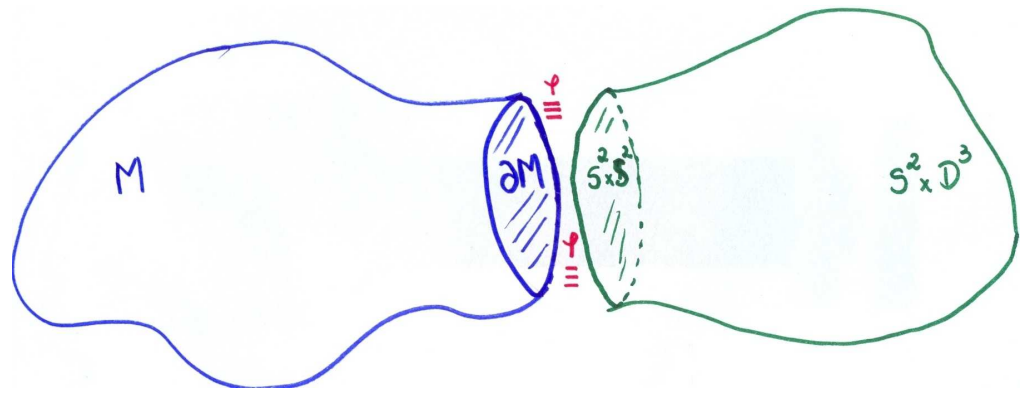

Sejam as funções inclusões $i: \psi\left(S^{2} \times S^{2}\right) \rightarrow M, j_{1}=i \circ \psi: S^{2} \times S^{2} \rightarrow M \mathrm{e}$ $j_{2}: S^{2} \times S^{2} \rightarrow S^{2} \times D^{3}$ e as classes $\xi_{1}=\left[S^{2} \times\{*\}\right]$ e $\xi_{2}=\left[\{*\} \times S^{2}\right]$.

Lema 2.2.5. Sejam $\xi_{1}=\left[S^{2} \times\{*\}\right], \xi_{2}=\left[\{*\} \times S^{2}\right] \in H_{2}\left(S^{2} \times S^{2}\right)$. Então $\left(j_{1}\right)_{*}\left(\xi_{1}\right)$ é um gerador de $H_{2}(M) \approx H_{2}\left(S^{2}\right) \approx \mathbb{Z}$.

Demonstração: Pelo teorema 2.2.3 $\left(j_{1}\right)_{*}\left(\xi_{2}\right)=(i \circ \psi)_{*}\left(\xi_{2}\right)=i_{*} \circ \psi_{*}\left(\xi_{2}\right)=i_{*}(\gamma)=0$. Logo existe um 3-ciclo $(D, \partial D)$ em $(M, \partial M)$ tal que $\partial D$ é homólogo a $\{*\} \times S^{2}$ em $S^{2} \times S^{2}$. Do fato de $\xi_{1} \bullet \xi_{2}=1$ em $\partial M$ a menos de orientação e de $[\partial D]=\xi_{2}$ segue que o número interseção em $M[D, \partial D] \bullet\left(j_{1}\right)_{*}\left(\xi_{1}\right)= \pm \xi_{1} \bullet \xi_{2}= \pm 1$. Assim, $\left(j_{1}\right)_{*}\left(\xi_{1}\right) \in H_{2}(M)$ é uma classe não nula, pois caso contrário $[D, \partial D] \bullet\left(j_{1}\right)_{*}\left(\xi_{1}\right)=0$.

Além disso, $\left(j_{1}\right)_{*}\left(\xi_{1}\right)$ é primitivo, caso contrário, $[D, \partial D] \bullet\left(j_{1}\right)_{*}\left(\xi_{1}\right) \neq \pm 1$. Portanto $\left(j_{1}\right)_{*}\left(\xi_{1}\right)$ é gerador de $H_{2}(M) \approx \mathbb{Z}$.

Teorema 2.2.5. $X$ é uma 5-variedade fechada e simplesmente conexa com $H_{2}(X) \approx \mathbb{Z}$.

Demonstração: Primeiramente como $M$ e $S^{2} \times D^{3}$ são 5 -variedades fechadas com bordos difeomorfos é claro que $X$ é 5 -variedade fechada, pois colamos os bordos de $M$ e $S^{2} \times D^{3}$. Lembremos que $M$ e $S^{2} \times D^{3}$ são conexos por caminho. Além disso, $\partial M$ e $S^{2} \times S^{2} \subset S^{2} \times D^{3}$ são subvariedades compactas e portanto existem $B_{1}$ e $B_{2}$ vizinhanças colarinhos de $\partial M$ em $M$ e de $S^{2} \times S^{2}$ em $S^{2} \times D^{3}$, respectivamente. Essas vizinhanças colarinhos dão origem a uma vizinhança bicolar $V$ de $\partial M \equiv_{\psi} S^{2} \times S^{2}$ em $X$ e, portanto, $V$ é difeomorfo a $\left(S^{2} \times S^{2}\right) \times(-\epsilon, \epsilon)$. Logo $V$ é conexo por caminho também. 
Consideremos os conjuntos $A=\left(M \cup_{\psi} B_{2}\right), C=\left(S^{2} \times D^{3} \cup_{\psi} B_{1}\right)$ e $(V=A \cap C)$, os quais são abertos em $X$ tal que $A \cup C=X$. Como $A$ se retrai em $M$ e $C$ se retrai em $S^{2} \times D^{3}$ segue que $\pi_{1}(A) \approx \pi_{1}(M) \approx\{1\}$ e $\pi_{1}(C) \approx \pi_{1}\left(S^{2} \times D^{3}\right) \approx \pi_{1}\left(S^{2}\right) \times \pi_{1}\left(D^{3}\right) \approx\{1\}$.

Portanto pelo teorema $1.1 .1 \pi_{1}(X) \approx\{1\}$.

Observe a seguinte sequência exata de Mayer-Vietoris de homologia:

$$
H_{2}\left(S^{2} \times S^{2}\right) \stackrel{\theta}{\rightarrow} H_{2}(M) \oplus H_{2}\left(S^{2} \times D^{3}\right) \stackrel{j=\left(j_{1}\right)_{*}-\left(j_{2}\right)_{*}}{\longrightarrow} H_{2}(X) \stackrel{k}{\rightarrow} H_{1}\left(S^{2} \times S^{2}\right) .
$$

onde $\theta=\left(\left(j_{1}\right)_{*},-\left(j_{2}\right)_{*}\right)$. A qual equivale a

$$
\mathbb{Z} \oplus \mathbb{Z} \stackrel{\theta}{\rightarrow} \mathbb{Z} \oplus \mathbb{Z}^{j=\left(\left(j_{1}\right)_{*}-\left(j_{2}\right)_{*}\right)} H_{2}(X) \stackrel{k}{\longrightarrow} 0
$$

Pelo lema 2.2.5, $\left(j_{1}\right)_{*}\left(\xi_{1}\right)$ é um gerador de $H_{2}(M)$ e já temos $\left(j_{2}\right)_{*}\left(\xi_{1}\right)$ é gerador de $H_{2}\left(S^{2} \times D^{3}\right)$ e $\left(j_{1}\right)_{*}\left(\xi_{2}\right)=\left(j_{2}\right)\left(\xi_{2}\right)=0$.

Como $\xi_{1}$ e $\xi_{2}$ são geradores de $H_{2}\left(S^{2} \times S^{2}\right)$ segue que a imagem de $\theta$, denotado por $\operatorname{Im}(\theta)$, é o conjunto $\left\{m\left(\left(j_{1}\right)_{*}\left(\xi_{1}\right),\left(j_{2}\right)_{*}\left(\xi_{1}\right)\right) ; m \in \mathbb{Z}\right\}$. É claro que $\operatorname{Im}(\theta)$ é isomorfo a $\mathbb{Z}$.

Pela sequência acima, $j$ é sobrejetiva e o núcleo de $j$, denotado por $\operatorname{Ker}(j)$, é igual a $\operatorname{Im}(\theta)$. Como o rank de $\mathbb{Z} \oplus \mathbb{Z}$ é 2 e o $\operatorname{Ker}(j)$ é isomorfo a $\mathbb{Z}$ segue que $H_{2}(X) \approx \mathbb{Z}$.

Proposição 2.2.3. Sejam as funções inclusões $j_{3}: M \rightarrow X, j_{4}: S^{2} \times D^{3} \rightarrow X$. Então $\left(j_{3}\right)_{*}: H_{2}(M) \rightarrow H_{2}(X)$ e $\left(j_{4}\right)_{*}: H_{2}\left(S^{2} \times D^{3}\right) \rightarrow H_{2}(X)$ são isomorfismos.

Demonstração: Observemos que os conjuntos $\left\{\left(\left(j_{1}\right)_{*}\left(\xi_{1}\right),\left(j_{2}\right)_{*}\left(\xi_{1}\right)\right),\left(\left(j_{1}\right)_{*}\left(\xi_{1}\right), 0\right)\right\}$ e $\left\{\left(\left(j_{1}\right)_{*}\left(\xi_{1}\right),\left(j_{2}\right)_{*}\left(\xi_{1}\right)\right),\left(0,\left(j_{2}\right)_{*}\left(\xi_{1}\right)\right)\right\}$ são bases para $H_{2}(M) \oplus H_{2}\left(S^{2} \times D^{3}\right)$. Portanto, como $j$ da sequência de Mayer-Vietoris do teorema acima é sobrejetiva, $j\left(\left(\left(j_{1}\right)_{*}\left(\xi_{1}\right),\left(j_{2}\right)_{*}\left(\xi_{1}\right)\right)\right)-$ $j\left(\left(\left(j_{1}\right)_{*}\left(\xi_{1}\right), 0\right)\right)$ é gerador de $H_{2}(X)$. Logo $\left(j_{3}\right)_{*}$ é isomorfismo. Com um argumento similar, $\left(j_{4}\right)_{*}$ também é isomorfismo.

Proposição 2.2.4. A segunda classe de Stiefel-Whitney de $X, w_{2}(X) \in H^{2}\left(X ; \mathbb{Z}_{2}\right)$, é nula. 
Demonstração: Lembremos que $\partial M \cong S^{2} \times S^{2}$. Assim $H^{2}\left(\partial M ; \mathbb{Z}_{2}\right) \approx H^{2}\left(S^{2} \times\right.$ $\left.S^{2} ; \mathbb{Z}_{2}\right) \approx 0$ e automaticamente $\partial M$ é uma variedade spin. Como $H^{1}\left(S^{2} \times S^{2} ; \mathbb{Z}_{2}\right) \approx$ $H^{1}\left(S^{2} ; \mathbb{Z}_{2}\right) \oplus H^{1}\left(S^{2} ; \mathbb{Z}_{2}\right) \approx 0$ tem-se uma única estrutura spin de $S^{2} \times S^{2}$.

Pela dualidade de Poincaré-Lefschetz

$$
H^{2}\left(M, \partial M ; \mathbb{Z}_{2}\right) \approx H_{5-2}\left(M ; \mathbb{Z}_{2}\right)=H_{3}\left(M ; \mathbb{Z}_{2}\right) \approx H_{3}\left(S^{2} ; \mathbb{Z}_{2}\right) \approx 0
$$

e

$$
H^{2}\left(S^{2} \times D^{3}, \partial\left(S^{2} \times D^{3}\right) ; \mathbb{Z}_{2}\right) \approx H_{5-2}\left(S^{2} \times D^{3} ; \mathbb{Z}_{2}\right)=H_{3}\left(S^{2} \times D^{3} ; \mathbb{Z}_{2}\right) \approx 0
$$

Portanto as classe relativas de Stiefel-Whitney $w_{2}(M, \partial M) \in H^{2}\left(M, \partial M ; \mathbb{Z}_{2}\right)$ e $w_{2}\left(S^{2} \times\right.$ $\left.D^{3}, \partial\left(S^{2} \times D^{3}\right)\right) \in H^{2}\left(S^{2} \times D^{3}, \partial\left(S^{2} \times D^{3}\right) ; \mathbb{Z}_{2}\right)$ são nulas.

De acordo com a prova de $4.3 \mathrm{em} \mathrm{[2],} w_{2}(X)=k_{*}\left(\left(w_{2}(M, \partial M), w_{2}\left(S^{2} \times D^{3}, \partial\left(S^{2} \times\right.\right.\right.\right.$ $\left.\left.\left.D^{3}\right)\right)\right)=k_{*}(0,0)=0$, onde $k_{*}: H^{2}\left(M, \partial M ; \mathbb{Z}_{2}\right) \oplus H^{2}\left(S^{2} \times D^{3}, \partial\left(S^{2} \times D^{3}\right) ; \mathbb{Z}_{2}\right) \rightarrow$ $H^{2}\left(X ; \mathbb{Z}_{2}\right)$ é homomorfismo que está melhor descrito neste artigo do Boyer.

Portanto, pelos resultados obtidos acima, $X$ é uma 5-variedade fechada, simplesmente conexa tal que a segunda classe de Stiefel-Whitney é igual a zero, isto é, $w_{2}(X)=0$ e $\operatorname{com} H_{2}(X) \approx \mathbb{Z}$.

Dessa forma pelos resultados do artigo [28] temos $X$ difeomorfo a $S^{2} \times S^{3}$. Assim como $X \cong S^{2} \times S^{3}$, então existe $W$ uma 6 -variedade compacta difeomorfo a $S^{2} \times D^{4}$ tal que $\partial W=X$. Denotaremos por $k: X \rightarrow W$ a função inclusão.

Teorema 2.2.6. As funções inclusões $k \circ j_{3}: M \rightarrow W e k \circ j_{4}: S^{2} \times D^{3} \rightarrow W$ são equivalências de homotopia.

Demonstração: Como vimos na proposição 2.2.3, as funções $\left(j_{3}\right)_{*}: H_{2}(M) \rightarrow H_{2}(X)$ e $\left(j_{4}\right)_{*}: H_{2}\left(S^{2} \times D^{3}\right) \rightarrow H_{2}(X)$ são isomorfismos.

Temos o seguinte diagrama comutativa 


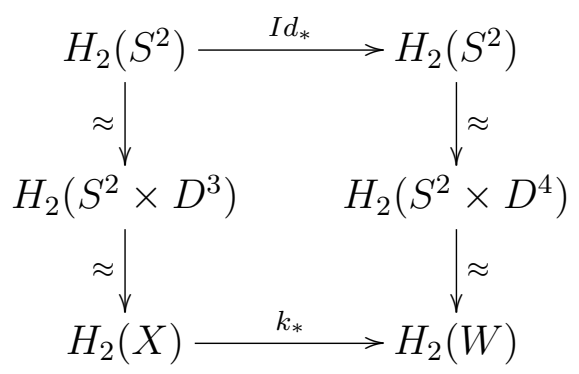

onde todas as setas verticais e $I d_{*}$ são isomorfismos, portanto, $k_{*}$ é isomorfismo.

$\operatorname{Logo}\left(k \circ j_{3}\right)_{*}: H_{2}(M) \rightarrow H_{2}(W)$ e $\left(k \circ j_{4}\right)_{*}: H_{2}\left(S^{2} \times D^{3}\right) \rightarrow H_{2}(W)$ também são isomorfismo.

Desde que $H_{*}(M) \approx H_{*}\left(S^{2}\right) \approx H_{*}\left(S^{2} \times D^{3}\right) \approx H_{*}(X)$ e $H_{*}(M) \approx H_{*}\left(S^{2}\right) \approx H_{*}\left(S^{2} \times\right.$ $\left.D^{4}\right) \approx H_{*}(W)$ segue que $k_{*}: H_{*}(X) \rightarrow H_{*}(W)$ é isomorfismo para todo $* \in \mathbb{Z}_{+}$e portanto $\left(k \circ j_{3}\right)_{*}: H_{*}(M) \rightarrow H_{*}(W)$ e $\left(k \circ j_{4}\right)_{*}: H_{*}\left(S^{2} \times D^{3}\right) \rightarrow H_{*}(W)$ também são isomorfismos para todo $* \in \mathbb{Z}_{+}$.

Do fato de $\pi_{1}(W) \approx \pi_{1}\left(S^{2} \times D^{4}\right) \approx \pi_{1}\left(S^{2}\right) \times \pi_{1}\left(D^{4}\right) \approx 0$ e $\pi_{1}(M) \approx \pi_{1}(X) \approx 0$ segue, pelo teorema 1.3.1 que $k \circ j_{3}$ e $k \circ j_{4}$ são equivalências de homotopias.

Seja $c: \partial\left(S^{2} \times D^{3}\right) \times[0,1] \rightarrow S^{2} \times D^{3}$ uma vizinhança colarinho de $\partial\left(S^{2} \times D^{3}\right)$ em $S^{2} \times D^{3} \operatorname{com} c\left(\partial\left(S^{2} \times D^{3}\right) \times\{0\}\right)=\partial\left(S^{2} \times D^{3}\right)$, a qual existe pelo teorema da vizinhança colarinho.

Sabemos que $\pi_{1}(W) \approx \pi_{1}(M) \approx\{1\}$. Consideremos o subconjunto $Y=c\left(\partial\left(S^{2} \times D^{3}\right) \times\right.$ $[0,1 / 2))$. O espaço $S^{2} \times D^{3}-Y$ é difeomorfo a $S^{2} \times D^{3}$ e, portanto, $\pi_{1}\left(S^{2} \times D^{3}-Y\right) \approx\{1\}$.

Portanto a tríade $\left(W, M, S^{2} \times D^{3}-Y\right)$ é um h-cobordismo. Além disso, $\operatorname{dim} W=6$.

Logo pelo Teorema do h-cobordismo, temos $W \cong M \times[0,1]$.

Concluindo assim que $M \cong S^{2} \times D^{3}-Y \cong S^{2} \times D^{3}$. Dessa forma fica provado o teorema abaixo.

Teorema 2.2.7. Seja $M$ uma 4-variedade compacta e simplesmente conexa satisfazendo as seguintes propriedades:

(i) $\partial M$ é difeomorfo a $S^{2} \times S^{2}$, 
(ii) $H_{*}(M) \approx H_{*}\left(S^{2}\right)$.

Então $M$ é difeomorfo a $S^{2} \times D^{3}$

Caso em que $p=q>2$

Lembremos que $M$ é uma $(2 \mathrm{p}+1)$-variedade compacta e simplesmente conexa satisfazendo:

(1) $\partial M \cong S^{p} \times S^{p}$

(2) $H_{*}(M) \approx H_{*}\left(S^{p}\right)$.

Seja $\delta \in H_{p}(\partial M)$ a classe de homologia citada no lema 2.2.4. Como $\partial M$ é simplesmente conexa, pois, $\pi_{1}(\partial M) \approx \pi_{1}\left(S^{p} \times S^{p}\right) \approx \pi_{1}\left(S^{p}\right) \times \pi_{1}\left(S^{p}\right) \approx\{1\}$ e $H_{i}(\partial M) \approx H_{i}\left(S^{p} \times\right.$ $\left.S^{p}\right) \approx 0$ para $i \in\{1, \ldots, p-1\}$, segue, pelo Teorema de Hurewicz, que $\pi_{p}(\partial M) \approx H_{p}(\partial M)$. Isto significa que para toda classe $\alpha \in H_{p}(\partial M)$ existe, através do isomorfismo, uma classe $\beta \in \pi_{p}(\partial M)$ que são relacionadas, isto é, para cada classe $\alpha \in H_{p}(\partial M)$ existe uma função contínua $\phi: S^{p} \rightarrow \partial M$ tal que $\phi_{*}\left(\left[S^{p}\right]\right)=\alpha$, onde $\left[S^{p}\right] \in H_{p}\left(S^{p}\right)$ é uma classe fundamental. Em particular, existe uma função contínua $\phi: S^{p} \rightarrow \partial M$ tal que $\phi_{*}\left(\left[S^{p}\right]\right)=\delta$.

Por [35] e [10] tem-se a existência de um mergulho $\phi^{\prime}: S^{p} \rightarrow \partial M$ tal que $\phi$ é homotópico a $\phi^{\prime}$.

Seja $S$ a subvariedade de $M$ a qual é obtida empurrando $\phi^{\prime}\left(S^{p}\right)$ para o interior de $M$, através do campo vetorial normal de $\partial M$ em $M$ apontando para dentro de $M$, isto é, para o interior de $M$.

Teorema 2.2.8. O fibrado normal de $S$ em $M$ é trivial.

Demonstração: Pelo teorema da vizinhança colarinho existe uma vizinhança colarinho de $\partial M$ em $M$. Seja $c^{\prime}: \partial M \times[0,1] \rightarrow M$ uma vizinhança colarinho de $\partial M$ em $M$, tal que $c^{\prime}(\partial M \times\{0\})=\partial M$.

Como $S$ está no interior de $M$, pode-se considerar $S$ no interior de $\operatorname{Im}\left(c^{\prime}\right)$. Sabemos que $\partial M \cong S^{p} \times S^{p}$ pode ser mergulhado em $\mathbb{R}^{2 p+1}$. Considere $\partial M$ como o bordo de uma vizinhança tubular de uma p-esfera mergulhada usualmente em $\mathbb{R}^{2 p+1}$. Dessa forma $\partial M \times[0,1]$ pode ser mergulhado em $\mathbb{R}^{2 p+1}$. 
Portanto o fibrado normal de $S$ em $M$ é equivalente ao fibrado normal de um mergulho de uma p-esfera em $\mathbb{R}^{2 p+1}$. Por outro lado, tem-se que o fibrado normal de um mergulho de uma q-esfera em $\mathbb{R}^{r}$ é trivial, desde que $\mathrm{r}-\mathrm{q}>\mathrm{q}$. Como $2 p+1-p=p+1>p$, o fibrado normal de $S$ em $M$ é trivial.

Agora considere $G$ uma vizinhança tubular de $S$ contida no interior de $M$ e sua função inclusão, denotada por $l: G \rightarrow M$. Como $S \cong S^{p}$ e o seu fibrado normal é trivial segue que $G$ é difeomorfo a $S^{p} \times D^{p+1}$.

Teorema 2.2.9. Dada l $: G \rightarrow M$ a função inclusão. Então a induzida em homologia $l_{*}: H_{i}(G) \rightarrow H_{i}(M)$ é um isomorfismo para todo $i \in\{0,1, \ldots\}$

Demonstração: Notemos que para todo $i \neq p$ e $i \neq 0, H_{i}(G)=H_{i}(M)=0$ e neste caso $l_{*}$ é um isomorfismo. No caso em que $i=0$, é claro que $l_{*}$ é isomorfismo.

Tem-se que $G$ é uma vizinhança tubular trivial de $S$. Portanto $H_{p}(G)$ é gerado pela classe $[S]$, que representa $S$. Por construção, $S$ é isotópica a $\phi^{\prime}\left(S^{p}\right)$ em $M$. Seja $i: \partial M \rightarrow$ $M$ a inclusão.

Pelo teorema 2.2.3, $i_{*}(\gamma)=0$ em $H_{p}(M)$. Portanto, existe um $(p+1)$-ciclo $(D, \partial D)$ em $(M, \partial M)$ tal que $\partial D$ é homólogo a $\gamma$ em $\partial M$, isto é, $[\partial D]=\gamma$ em $\partial M$. Logo o número interseção $[D, \partial D] \bullet i_{*}(\delta)$ em $M$ é igual, a menos de sinal, ao número interseção $\gamma \bullet \delta$ em $\partial M$, onde $\delta$ é como no teorema 2.2.4.

Pelo teorema $2.2 .4 \gamma \bullet \delta=1$. Assim, $[D, \partial D] \bullet i_{*}(\delta)= \pm 1$.

Portanto, $i_{*}(\delta)$ não é nula e primitiva de $H_{p}(M) \approx \mathbb{Z}$. Logo $l_{*}$ é sobrejetiva.

Tomemos o conjunto $V=M-i n t G$. Como $\operatorname{dim} S=p$ e portanto a codimensão de $S$ é $p+1 \geq 4$, temos $\pi_{1}(V) \approx \pi_{1}(M-$ Int $G) \approx \pi_{1}(M-S) \approx \pi_{1}(M) \approx\{1\}$.

Considere $M-\operatorname{Int}(G), G-\operatorname{Int}(G), M-K^{\prime}$ e $G-K^{\prime}$, onde $K^{\prime}$ é um espaço difeomorfo a $\operatorname{Int}(G)$ de forma que $K^{\prime} \subset \operatorname{Int}(G), G-K^{\prime} \cong G-\operatorname{Int}(G)$ e $M-\operatorname{Int}(G) \cong M-K^{\prime}$. $\left(\right.$ Como $G \cong S^{p} \times D^{p+1}$ então $\operatorname{Int}(G) \cong S^{p} \times B^{p+1}$, assim basta diminuir o raios de $B^{p+1}$ e tomar $K^{\prime}$ o conjunto difeomorfo. Logo existe $K^{\prime}$ ). Assim $\overline{K^{\prime}} \subset G$. 
Portanto, aplicando o Teorema da Excisão tem-se $H_{*}(M-\operatorname{Int}(G), G-\operatorname{Int}(G)) \approx$ $H_{*}\left(M-K^{\prime}, G-K^{\prime}\right) \approx H_{*}(M, G)$.

Pela sequência de homologia do par $(M, G)$, tem-se os seguintes casos:

(i) Quando $r \neq 0,1, p, p+1$

$$
H_{r}(G) \approx 0 \rightarrow H_{r}(M) \approx 0 \rightarrow H_{r}(M, G) \rightarrow H_{r-1}(G) \approx 0
$$

Logo, $H_{r}(M, G) \approx 0$.

(ii) Quando $r=0$

$$
H_{0}(G) \stackrel{\approx_{l_{*}}}{\rightarrow} H_{0}(M) \rightarrow H_{0}(M, G) \rightarrow 0
$$

Logo, $H_{0}(M, G) \approx 0$.

(iii) Quando $r=1$

$$
H_{1}(G) \approx 0 \rightarrow H_{1}(M) \approx 0 \rightarrow H_{1}(M, G) \rightarrow H_{0}(G) \stackrel{\approx_{l_{*}}}{\rightarrow} H_{0}(M)
$$

Logo, $H_{1}(M, G) \approx 0$.

(iv) Quando $r=p$

$$
H_{p}(G) \stackrel{\approx_{l_{*}}}{\rightarrow} H_{p}(M) \rightarrow H_{p}(M, G) \rightarrow H_{p-1}(G) \approx 0
$$

Logo, $H_{p}(M, G) \approx 0$.

E finalmente,

(v) Quando $r=p+1$

$$
H_{p+1}(G) \approx 0 \rightarrow H_{p+1}(M) \approx 0 \rightarrow H_{p+1}(M, G) \rightarrow H_{p}(G) \stackrel{\approx_{l_{*}}}{\rightarrow} H_{p}(M)
$$

$\operatorname{Logo}, H_{p+1}(M, G) \approx 0$.

Portanto, segue-se que $H_{*}(M, G) \approx 0$.

Sendo que $\pi_{1}(V) \approx \pi_{1}(\partial M) \approx \pi_{1}(G) \approx\{1\}$ e $H_{*}(M, G) \approx 0$, do teorema 1.5.6 segue-se que $V \cong \partial G \times[0,1]$. 
Logo, $M=G \cup V$ e assim,

$$
M \cong S^{p} \times D^{p+1} \cup \partial\left(S^{p} \times D^{p+1}\right) \times[0,1] \cong S^{p} \times D^{p+1} \cup\left(S^{p} \times S^{p}\right) \times[0,1] \cong S^{p} \times D^{p+1}
$$

Dessa forma temos provado o seguinte teorema:

Teorema 2.2.10. Seja uma $(2 p+1)$-variedade $M$ compacta, simplesmente conexa com as seguintes propriedades:

(i) $p \geq 2$,

(ii) $\partial M$ é difeomorfo a $S^{p} \times S^{p}$,

(iii) $H_{*}(M) \approx H_{*}\left(S^{p}\right)$.

Então $M$ é difeomorfa a $S^{p} \times D^{p+1}$.

\subsubsection{Caso em que $p>q=1$}

Lembre que:

(i) $f: S^{p} \times S^{1} \rightarrow S^{p+2}$ é um mergulho suave.

(ii) $C_{1}$ é a componente conexa por caminho de $S^{p+2}-f\left(S^{p} \times S^{1}\right)$ a qual tem a homologia de $S^{p}$.

Nestas hipóteses, tem-se o seguinte teorema;

Teorema 2.2.11. Quando $q=1$ e $p \geq 2, C_{1}$ é uma componente simplesmente conexa.

Demonstração: Seja a inclusão $i_{2}: \partial C_{2} \rightarrow C_{2}$. Similarmente ao teorema 2.2 .2 , prova-se que $\left(i_{2}\right)_{*}: H_{1}\left(\partial C_{2}\right) \rightarrow H_{1}\left(C_{2}\right)$ é isomorfismo. Lembre que pelo teorema 1.2.2, $H_{1}\left(\partial C_{2}\right) \approx \frac{\pi_{1}\left(\partial C_{2}\right)}{C}$, onde $C$ é o subgrupo comutador de $\pi_{1}\left(C_{2}\right)$.

$\operatorname{Mas} \pi_{1}\left(\partial C_{2}\right) \approx \pi_{1}\left(f\left(S^{p} \times S^{1}\right)\right) \approx \pi_{1}\left(S^{p} \times S^{1}\right) \approx \mathbb{Z}$. Portanto $H_{1}\left(\partial C_{2}\right) \approx \pi_{1}\left(\partial C_{2}\right)$.

Agora consideremos as inclusões $i_{1}: \partial C_{1} \rightarrow C_{1}, i_{2}: \partial C_{2} \rightarrow C_{2}, \bar{i}_{1}: C_{1} \rightarrow S^{p+2}$, $\bar{i}_{2}: C_{2} \rightarrow S^{p+2}$ e $i: \partial C_{1} \rightarrow S^{p+2}$ com o seguinte diagrama comutativo 


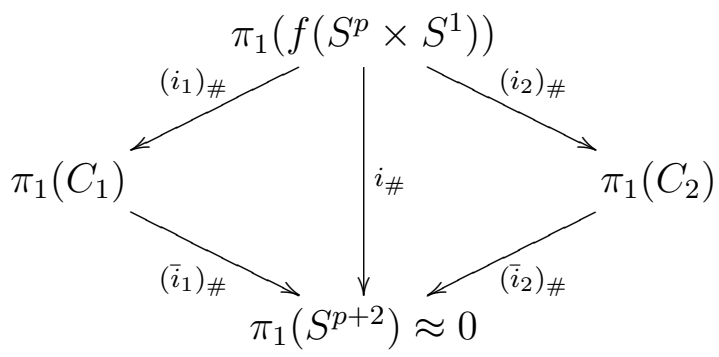

Além disso, pelo corolário 1.2 .2 temos o seguinte diagrama comutativo

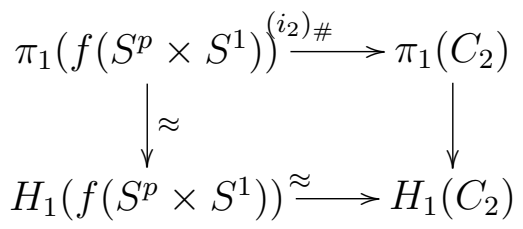

e, portanto, $\left(i_{2}\right)_{\#}$ é injetiva.

Suponha que $\left(i_{1}\right)_{*}$ seja injetiva também. Assim, pelo corolário 1.1.1.1, $\left(\bar{i}_{2}\right)_{\#}$ e $\left(\bar{i}_{1}\right)_{\#}$ seriam injetivas e, portanto, $i_{*}$ também o é.

Isto implica que $i_{*}\left(\pi_{1}\left(f\left(S^{p} \times S^{1}\right)\right)\right) \approx \pi_{1}\left(f\left(S^{p} \times S^{1}\right)\right) \approx \pi_{1}\left(S^{p} \times S^{1}\right) \approx \mathbb{Z}$ é um subgrupo de $\pi_{1}\left(S^{p+2}\right) \approx\{1\}$. Mas isto é uma contradição pois $\pi_{1}\left(S^{p+2}\right) \approx\{1\}$ e, portanto, $\left(i_{1}\right)_{\#}$ não é injetiva.

Seja $t$ um gerador de $\pi_{1}\left(f\left(S^{p} \times S^{1}\right)\right)$ representando a classe de $f\left(\{*\} \times S^{1}\right)$. Como vimos que $\left(i_{1}\right)_{\#}$ não é injetiva, então existe um inteiro positivo $r^{\prime}$ tal que $\left(i_{1}\right)_{\#}\left(t^{r^{\prime}}\right)=1$. Denotemos por $r$ o menor inteiro positivo tal que $\left(i_{1}\right)_{\#}\left(t^{r}\right)=1$.

Considere os seguintes subgrupos

(i) $T$ é o subgrupo gerado por $t^{r}$, o qual é normal pois $\pi_{1}\left(f\left(S^{p} \times S^{1}\right) \approx \mathbb{Z}\right.$ é abeliano.

(ii) $N$ é o menor subgrupo normal de $\pi_{1}\left(C_{2}\right)$ que contenha o subgrupo gerado por $y=$ $\left(i_{2}\right)_{\#}\left(t^{r}\right)$, isto é, os elementos de $N$ são da forma $x_{1} y^{ \pm 1} x_{1}^{-1} \ldots x_{m} y^{ \pm 1} x_{m}^{-1}$, para alguns $x_{i} \in \pi_{1}\left(C_{2}\right)$.

Sejam as seguintes funções $\alpha, \beta, \bar{\alpha}, \bar{\beta}$ e $\omega$ induzidas nos grupos quocientes abaixo $\left(i_{1}\right)_{\#},\left(i_{2}\right)_{\#},\left(\bar{i}_{1}\right)_{\#},\left(\bar{i}_{2}\right)_{\#}$ e $i_{\#}$, respectivamente, como segue no diagrama abaixo 


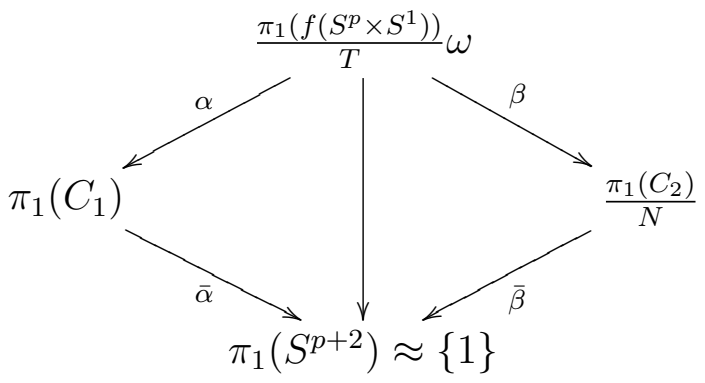

Note que pela definição de $r$ a função $\alpha$ é injetiva. Suponha que $\beta$ não seja injetiva. Então existe um inteiro positivo $0<s<r$ tal que $\beta\left(t^{s}+T\right)=0$, isto é, $\left(i_{2}\right)_{\#}\left(t^{s}\right) \in N$.

Pela definição de $N$, temos

$$
\left(i_{2}\right)_{\#}\left(t^{s}\right)=x_{1} y^{ \pm 1} x_{1}^{-1} \ldots x_{m} y^{ \pm 1} x_{m}^{-1} \text {, para alguns } x_{i} \in \pi_{1}\left(C_{2}\right) .
$$

Seja $H$ o grupo abelianizado de $\pi_{1}\left(C_{2}\right)$, então pelo corolário $1.2 .2 H \approx H_{1}\left(C_{2}\right) \approx \mathbb{Z}$.

Em $H$ tem-se $\left(i_{2}\right)_{\#}\left(t^{s}\right)=y^{e}=\left(\left(i_{2}\right)_{\#}\left(t^{r}\right)\right)^{e}$, para algum inteiro $e$.

Da mesma forma como foi feito no lema 2.2.2 que $H_{1}\left(C_{2}\right)$ é gerado pela classe $\left(i_{2}\right)_{\#}(t)$, lembrando que $t=\left[f\left(\{*\} \times S^{1}\right)\right]$.

Portanto, como $\left(i_{2}\right)_{\#}\left(t^{s}\right)=\left(\left(i_{2}\right)_{\#}\left(t^{r}\right)\right)^{e}$, tem-se $\left(\left(i_{2}\right)_{\#}(t)\right)^{s-r e}=0$ em $H$. O que implica que $s-r e=0$.

Mas isto contradiz o fato de $0<s<r$. Portanto o absurdo esta em supor que $\beta$ não é injetiva. Logo $\beta$ tem que ser injetiva.

Pelo corolário 1.1.1.1, sendo $\alpha$ e $\beta$ injetivas, tem-se que $\bar{\alpha}$ e $\bar{\beta}$ são injetivas. Logo $\omega$ também é injetiva.

Portanto, como $\pi_{1}\left(S^{p+2}\right) \approx\{1\}$ e $r$ é a ordem de $\frac{\pi_{1}\left(S^{p} \times S^{1}\right)}{T}, r= \pm 1$.

Finalmente, pelo teorema 1.1.1 e o diagrama acima, $\pi_{1}\left(S^{p+2}\right)$ é isomorfo ao produto livre de $\pi_{1}\left(C_{1}\right)$ e $\frac{\pi_{1}\left(C_{2}\right)}{N}$. Logo, como $\pi_{1}\left(S^{p+2}\right) \approx\{1\}, \pi_{1}\left(C_{1}\right)$ é trivial.

Guardemos a informação, citada acima, de que $\pi_{1}\left(C_{2}\right)$ é gerado por $\left(i_{2}\right)_{\#}(t)$.

Queremos provar o seguinte teorema:

Teorema 2.2.12. Seja $M$ uma $p+2$-variedade compacta, simplesmente conexa tal que satisfaz as seguintes condições: 
(i) $p \geq 2$

(ii) $\partial M$ é difeomorfo a $S^{p} \times S^{1}$

(iii) $H_{*}(M) \approx H_{*}\left(S^{p}\right)$.

Então se $p>2 M$ é difeomorfo a $S^{p} \times D^{2}$ e caso $p=2 M$ é homeomorfo a $S^{2} \times D^{2}$.

\section{Demonstração: Caso $p=2$}

Para este caso observemos que $H_{1}(\partial M) \approx H_{1}\left(S^{2} \times S^{1}\right) \approx \mathbb{Z}$ e, portanto, $H_{1}(\partial M)$ é livre de torção. Além disso, a forma interseção de $M$ é trivial e par.

Por [2], $\mathcal{H}_{+}\left(S^{1} \times S^{2}\right)$ atua transversalmente sobre $\operatorname{Spin}\left(S^{1} \times S^{2}\right)$ e conclui que a cardinalidade de $\nu_{L}(\partial M)$ é 1 , onde $\nu_{L}(\partial M)$ é o conjunto de todos as 4-variedades homeomorfas, compactas, simplesmente conexas, orientada com bordo igual a $\partial M$ e a forma interseção como de $M$. Portanto, como $S^{2} \times D^{2}$ tem como bordo $S^{2} \times S^{1}$, segue que $M$ é homeomorfa a $S^{2} \times D^{2}$.(Para os detalhes ver [2]).

Dividiremos a demonstração do outro caso, $p \geq 3$, em passos.

Caso $p \geq 3$

(i) passo 1: Como $\partial M$ é difeomorfo a $S^{p} \times S^{1}$, existe um difeomorfismo $\phi: S^{p} \times S^{1} \rightarrow \partial M$. Consideremos a função inclusão $j: \phi\left(S^{p} \times\{*\}\right) \rightarrow M$. Pelo mesmos argumentos do teorema 2.2 .2 segue que $j$ induz um isomorfismo $j_{*}: H_{p}\left(\phi\left(S^{p} \times\{*\}\right)\right) \rightarrow H_{p}(M)$ e como

$$
H_{i}\left(\phi\left(S^{p} \times\{*\}\right)\right) \approx H_{i}\left(S^{p} \times\{*\}\right) \approx H_{i}\left(S^{p}\right) \approx H_{i}(M) \approx 0, \forall i \neq 0, p
$$

e quando $i=0 j_{*}$ já é isomorfismo, tem-se $j_{*}$ isomorfismo para todo $i$.

Portanto, pelo teorema 1.3.1, $j$ é uma equivalência de homotopia, já que $\pi_{1}\left(\phi\left(S^{p} \times\right.\right.$ $\{*\})) \approx \pi_{1}\left(S^{p} \times\{*\}\right) \approx\{1\} \approx \pi_{1}(M)$.

(ii) passo2: Denotemos por $X=M \cup_{\phi} S^{p} \times D^{2}$, isto é, $X$ é a colagem ao longo do bordo de $M$ a $S^{p} \times D^{2}$ através da $\phi$.

Provemos algumas características de $X$ que são:

1) Da mesma forma como foi feito na prova do teorema 2.2.5, usando o teorema 1.1.1 e lembrando que $p \geq 3$, prova-se que $X$ é uma $p+2$-variedade fechada e simplesmente conexa.

2) Provemos que $H_{*}(X) \approx H_{*}\left(S^{p} \times S^{2}\right)$. 
Novamente usando os mesmos argumentos do teorema 2.2.5, temos a existência da seguinte sequência exata de Mayer-Vietoris

$$
\ldots \rightarrow H_{i}\left(S^{p} \times S^{1}\right) \rightarrow H_{i}(M) \oplus H_{i}\left(S^{p} \times D^{2}\right) \rightarrow H_{i}(X) \rightarrow H_{i-1}\left(S^{p} \times S^{1}\right) \rightarrow \ldots
$$

Analisemos os casos da sequência acima.

caso 1: $i \neq 0,1,2, p, p+1$ temos

$$
H_{i}\left(S^{p} \times S^{1}\right) \approx 0 \rightarrow H_{i}(M) \approx 0 \oplus H_{i}\left(S^{p} \times D^{2}\right) \approx 0 \rightarrow H_{i}(X) \rightarrow H_{i-1}\left(S^{p} \times S^{1}\right) \approx 0
$$

Portanto $H_{i}(X) \approx 0$

caso 2: $i=0$ temos

$$
H_{0}\left(S^{p} \times S^{1}\right) \longmapsto H_{0}(M) \oplus H_{0}\left(S^{p} \times D^{2}\right) \rightarrow H_{0}(X) \rightarrow 0 .
$$

Portanto $H_{0}(X) \approx \mathbb{Z}$

caso 3: $i=1$ temos

$$
H_{1}(M) \approx 0 \oplus H_{1}\left(S^{p} \times D^{2}\right) \approx 0 \rightarrow H_{1}(X) \rightarrow H_{0}\left(S^{p} \times S^{1}\right) \longmapsto H_{0}(M) \oplus H_{0}\left(S^{p} \times D^{2}\right) .
$$

Portanto $H_{1}(X) \approx 0$

caso 4: $i=2$ temos

$$
\begin{gathered}
H_{2}\left(S^{p} \times S^{1}\right) \approx 0 \rightarrow H_{2}(M) \approx 0 \oplus H_{2}\left(S^{p} \times D^{2}\right) \approx 0 \rightarrow \\
\rightarrow H_{2}(X) \oplus H_{1}\left(S^{p} \times S^{1}\right) \approx \mathbb{Z} \rightarrow H_{1}(M) \approx 0 \oplus H_{1}\left(S^{p} \times D^{2}\right) \approx 0 .
\end{gathered}
$$

Portanto $H_{2}(X) \approx \mathbb{Z}$.

caso 5: $i=p$ temos

$$
H_{p}\left(S^{p} \times S^{1}\right) \approx \mathbb{Z} \longmapsto H_{p}(M) \approx \mathbb{Z} \oplus H_{p}\left(S^{p} \times D^{2}\right) \approx \mathbb{Z} \rightarrow H_{p}(X) \rightarrow 0 .
$$


Portanto $H_{p}(X) \approx \mathbb{Z}$

caso 6: $i=p+1$ temos

$H_{p+1}(M) \approx 0 \oplus H_{p+1}\left(S^{p} \times D^{2}\right) \approx 0 \rightarrow H_{p+1}(X) \rightarrow H_{p}\left(S^{p} \times S^{1}\right) \approx \mathbb{Z} \mapsto H_{p}(M) \approx \mathbb{Z} \oplus H_{p}\left(S^{p} \times D^{2}\right) \approx \mathbb{Z}$

Portanto $H_{p+1}(X) \approx 0$

As injetividade dos casos 2), 3), 5), e 6) seguem do fato de $j$, definida acima, ser equivalência de homotopia.

Portanto, dos casos acima conclui-se que $H_{*}(X) \approx H_{*}\left(S^{p} \times S^{2}\right)$.

(iii) passo 3: Sejam os conjuntos $S_{1}=\Sigma_{1}=S^{p} \times\left\{*_{1}\right\}$ e $K=\Sigma_{2}=\left\{*_{2}\right\} \times S^{1}$ tal que $S_{1}$, $K \subset S^{p} \times S^{1}=\partial\left(S^{p} \times D^{2}\right)$. Observemos que, como $\pi_{1}\left(S^{p} \times D^{2}\right) \approx\{1\}, K$ borda um 2-disco suave $D_{1}=\left\{*_{2}\right\} \times D^{2}$ em $S^{p} \times D^{2}$.

Por outro lado, como $M$ é simplesmente conexa, existe um 2-disco em $M$ tal que o bordo é $\phi(K) \subset \partial M$, o qual pode não ser suave. Mas como a dimensão de $M$ é maior ou igual a 5, podemos tomar um 2-disco mergulhado suavemente por transversalidade (no artigo [10] encontra-se este resultado).

Denotemos por $D_{2}$ um 2-disco mergulhado tal que $\partial\left(D_{2}\right)=\phi(K)$.

Do fato de existir uma vizinhança colarinho de $\partial M$ em $M$, podemos assumir que $D_{2} \cap \partial M=\partial D_{2}$ e como $D_{2}$ foi construído, por transversalidade, $D_{2}$ é transversal a $\partial M$.

Do modo como $D_{1}$ e $D_{2}$ foram construídos, temos $S_{2}=D_{1} \cup D_{2}$ uma 2-esfera em $X$ e $S_{2}$ é transversal a $S_{1}$ no ponto $\left(*_{1}, *_{2}\right) \in \partial\left(S^{p} \times D^{2}\right)$.

Corolário 2.2.12.1. Seja a inclusão $j: S_{1} \cup S_{2} \rightarrow X$. Então $j$ induz isomorfismo a nível de homologia, isto é,

$$
j_{*}: H_{i}\left(S_{1} \cup S_{2}\right) \rightarrow H_{i}(X)
$$

é um isomorfismo quando $i \neq p+2$.

Demonstração: Pelo Passo 2) anterior foi provado que $H_{i}(X) \approx H_{i}\left(S^{p} \times S^{2}\right)$. Portanto, para os casos em que $i \neq 0,2, p, j_{*}$ é isomorfismo. Como $S_{1}$ e $S_{2}$ se interceptam num ponto e cada um desses é conexo por caminho segue-se que $S_{1} \cup S_{2}$ é conexo por 
caminho, $\operatorname{logo} j_{*}$ é isomorfismo para $i=0$. No caso $i=2$, foi visto que $S_{2} \cong S$, onde $S$ é uma 2-esfera, assim $j_{*}$ é isomorfismo. Também na demonstração de Passo 2) foi provado que $H_{p}(X)$ é gerado por $\left[S_{1}\right]$, ou seja, $j_{*}$ também é isomorfismo. Portanto, $j_{*}$ é isomorfismo para todo $i \neq p+2$.

Como visto na prova do lema 2.2.1, o fibrado normal $\nu_{1}$ de $S_{1}$ é trivial. Mas o fibrado normal de $S_{2}$ pode não ser trivial.

Agora lembremos que o grupo $\pi_{1}(S O(p+1))$, isto é, o grupo fundamental do grupo de matrizes $S O(p+1)\left(S O(p+1)\right.$ pode ser pensado como o grupo dos difeomorfismo de $S^{p}$ em $\left.S^{p}\right)$. Logo, o grupo $\pi_{1}(S O(p+1))$ é o conjunto dos difeomorfismos $\psi: S^{p} \times S^{1} \rightarrow S^{p} \times S^{1}$ passado ao quociente pela relação de homotopia.

De ([15], pag 97), o corolário da proposição 18, foi provado que se $n \geq 3$ então $\pi_{1}(S O(n)) \approx \mathbb{Z}_{2}$ e no nosso caso $p+1 \geq 4$ e, portanto, $\pi_{1}(S O(p+1)) \approx \mathbb{Z}_{2}$.

Assim, neste caso os fibrados normais da $S_{2}$, que é uma 2-esfera em $X$, é determinado pelo grupo fundamental $\pi_{1}(S O(p+1)) \approx \mathbb{Z}_{2}$ (Para isto ver resultados de fibrados no livro [30]).

Seja $\tau: S^{p} \times S^{1} \rightarrow S^{p} \times S^{1}$ o difeomorfismo o qual corresponde ao gerador de $\pi_{1}(S O(p+$ 1)) e satisfaça $\tau\left(\left\{*_{2}\right\} \times S^{1}\right)=\left\{*_{2}\right\} \times S^{1}$.

Tomemos $\phi^{\prime}=\phi \circ \tau$. Como $\phi$ e $\tau$ são difeomorfismo é claro que $\phi^{\prime}$ também o é.

Portanto, se caso, quando construímos $X$ através da $\phi$, o fibrado normal $\nu_{2}$ de $S_{2}$ não for trivial, então basta considerar $\phi^{\prime}$ para construir $X$, a qual satisfaz as mesmas condições que $\phi$. Dessa forma o fibrado normal $\nu_{2}$ de $S_{2}$ será trivial.

Logo, podemos assumir que o fibrado normal de $S_{2}$ é trivial.

Já que o fibrado normal é trivial temos vizinhanças tubulares $N_{1}$ e $N_{2}$ respectivamente de $S_{1}$ e $S_{2}$ em $X$ tal que $N_{1} \cong S^{p} \times D^{2}$ e $N_{2} \cong D^{p} \times S^{2}$. Portanto, $N_{1} \cup N_{2} \cong$ $S^{p} \times S^{2}-\operatorname{Int}\left(D^{p+2}\right)$, onde $\operatorname{Int}\left(D^{p+2}\right)$ é o interior de um $\mathrm{p}+2$-disco.

Teorema 2.2.13. Seja $\Delta=X-\operatorname{Int}\left(N_{1} \cup N_{2}\right)$. Então $\Delta$ é difeomorfo a um (p+2)-disco.

Demonstração: Lembremos que $S_{1}=S^{p} \times\left\{*_{1}\right\} \subset S^{p} \times S^{1}$ e $S_{2}=D_{2} \cup D_{1}$, onde $D_{1}=\left\{*_{2}\right\} \times D^{2} \subset S^{p} \times D^{2}$ e $D_{2}$ é um disco mergulhado em $M$ tal que $\partial D_{2}=\phi\left(S^{\prime}\right)$, onde 
$S^{\prime}=\left\{*_{1}\right\} \times S^{1} \subset S^{p} \times S^{1}$.

Como $S_{1}$ está no bordo de $S^{p} \times D^{2}$ e $S_{1}$ está no bordo de $M$ com $S^{p} \times D^{2}$ e $M$ simplesmente conexos. Segue, usando 1.1.1, que $S^{p} \times D_{2}-S_{1}$ e $M-S_{1}$ são simplesmente conexos também.

Lembre que a codimensão de $D_{1}$ em $S^{p} \times D^{2}$ é maior ou igual a 3. Além disso a codimensão de $D_{2}$ em $M$ também é maior ou igual a 3 . Portanto, segue-se que $\pi_{1}\left(S^{p} \times\right.$ $\left.D^{2}-\left(S_{1} \cup D_{1}\right)\right) \approx \pi_{1}\left(M-\left(S_{1} \cup D_{2}\right)\right) \approx\{1\}$.

Usando as mesmas técnicas da demonstração do teorema 2.2.5, através do teorema 1.1.1, segue que $M-\operatorname{Int}\left(N_{1} \cup N_{2}\right)$ e $S^{p} \times D^{2}-\operatorname{Int}\left(N_{1} \cup N_{2}\right)$ são simplesmente conexos, pois $N_{1}$ e $N_{2}$ são vizinhanças tubulares triviais.

Pelo teorema 1.1.1, $\Delta$ é simplesmente conexo, pois $\left(M \cap S^{p} \times D^{2}\right)-\operatorname{Int}\left(N_{1} \cup N_{2}\right)$ é conexo.

Pela dualidade de Poincaré-Lefschetz temos

$$
H_{i}(\Delta) \approx H^{p+2-i}(\Delta, \partial \Delta)
$$

Da mesma forma, pelo Teorema da Excisão,

$$
H^{p+2-i}(\Delta, \partial \Delta) \approx H^{p+2-i}\left(X, N_{1} \cup N_{2}\right)
$$

Sendo $N_{i}$ uma vizinhança tubular de $S_{i}, i=1,2$, tem-se

$$
H^{p+2-i}\left(X, N_{1} \cup N_{2}\right) \approx H^{p+2-i}\left(X, S_{1} \cup S_{2}\right)
$$

Pelo teorema 2.2.12.1, $H^{i}\left(X, S_{1} \cup S_{2}\right) \approx 0, \forall i>0$. Logo, $\Delta$ é acíclico. Como $\Delta$ é acíclico e simplesmente conexo, segue-se que $\Delta$ é contrátil.

Como $p+1 \geq 3$, pela conjectura de Poincaré, $\partial D$ é difeomorfo a uma $(p+1)$-esfera. Portanto $\delta$ é difeomorfo a um $(p+2)$-disco.

Relembremos que $N_{1} \cup N_{2} \cong S^{p} \times S^{2}-\operatorname{Int}\left(D^{p+2}\right)$. Portanto como $\Delta$ é difeomorfo a um $(p+2)$-disco, temos $X$ difeomorfo a colagem de um $(p+2)$-disco a $N_{1} \cup N_{2}$ ao longo 
do bordo, isto é, $X$ é a soma conexa $S^{p} \times S^{2} \sharp \Sigma$, onde $\Sigma$ é uma $(p+2)$-esfera. Mudando $\phi$ adequadamente no $(p+1)$-disco em $\partial\left(S^{p} \times D^{2}\right)$, tem-se $X$ difeomorfo a $S^{p} \times S^{2}$.

Portanto, $X$ borda uma $(p+3)$ - variedade compacta $W$ difeomorfa a $S^{p} \times D^{3} \cong$ $S^{p} \times D^{2} \times I$.

Teorema 2.2.14. Sejam as inclusões $i: M \rightarrow W$ e $j: S^{p} \times D^{2} \rightarrow W$. Então ambas são equivalências de homotopia.

Demonstração: Como na demonstração do teorema 2.2.5, e usando a sequência exata de Mayer-Vietoris prova-se que

$$
H_{i}(M) \approx H_{i}(X) \approx H_{i}\left(S^{p} \times D^{3}\right)
$$

Da mesma forma que foi feito no teorema 2.2.6, usando o teorema 1.3.1, prova-se que $i$ e $j$ são equivalências de homotopia, já que $\pi_{1}(M) \approx \pi_{1}\left(S^{p}\right) \approx \pi_{1}\left(S^{p} \times D^{2}\right) \approx\{1\}$ e $\pi_{1}(W) \approx \pi_{1}\left(S^{p} \times D^{3}\right) \approx\{1\}$.

Quando $p \geq 2$ e $q=1$, pelo teorema 2.2.11, $C_{1}$ é uma $p+2$-variedade suave simplesmente conexa cuja homologia é a mesma que $S^{p}$ e seu bordo é difeomorfo a $S^{p} \times S^{1}$. Portanto para mostrar que quando $p>2$ e $q=1, C_{1}$, é difeomorfo a $S^{p} \times D^{2}$ e quando $p=2$ e $q=1, C_{1}$ é homeomorfo a $S^{2} \times D^{2}$, basta mostrar o seguinte teorema:

Teorema 2.2.15. Seja $M$ uma $(p+2)$-variedade compacta, simplesmente conexa com as seguintes propriedades:

(i) $p \geq 2$

(ii) $\partial M$ é difeomorfo a $S^{p} \times S^{1}$.

(iii) $H_{*}(M) \approx H_{*}\left(S^{p}\right)$.

\section{Então}

(1) se $p>2, M$ é difeomorfo a $S^{p} \times D^{2}$,

(2) se $p=2, M$ é homeomorfo a $S^{2} \times D^{2}$.

Demonstração: Lembre que no caso $p=2$ já foi provado. 
Para o caso $p>2$ considere $W$ o espaço construído anteriormente. Seja $c: \partial\left(S^{p} \times\right.$ $\left.D^{2}\right) \times[0,1] \rightarrow S^{p} \times D^{2}$ uma vizinhança colarinho de $\partial\left(S^{p} \times D^{2}\right) \operatorname{com} c\left(\partial\left(S^{p} \times D^{2}\right) \times\{0\}\right)=$ $\partial\left(S^{p} \times D^{2}\right)$.

Tomemos a tripla $\left(W, M, S^{p} \times D^{2}-Y\right)$, onde $Y=c\left(\partial\left(S^{p} \times D^{2}\right) \times\left[0, \frac{1}{2}\right)\right)$. O espaço $S^{p} \times D^{2}-Y$ se retrai em $S^{p} \times D^{2}$, logo é difeomorfo a $S^{p} \times D^{2}$ e, portanto, $\pi_{1}\left(S^{p} \times D^{2}-Y\right) \approx$ $\pi_{1}\left(S^{p} \times D^{2}\right) \approx\{1\}$.

Além disso $\pi_{1}(W) \approx \pi_{1}(M) \approx\{1\}$ e $\operatorname{dim}(W) \geq 6$.

Portanto a tríade $\left(W, M, S^{p} \times D^{2}-Y\right)$ é um h-cobordismo. Logo, pelo teorema do h-cobordismo, temos $W \cong M \times[0,1]$ e com isso temos $M \cong S^{p} \times D^{2}$.

Resumindo para quaisquer $p$ e $q$, tem-se o seguinte resultado.

Teorema 2.2.16. Sejam $f: S^{p} \times S^{q} \rightarrow S^{p+q+1}$ um mergulho suave e os fechos das componentes conexas por caminhos de $S^{p+q+1}-f\left(S^{p} \times S^{q}\right)$.

1) se $p \geq q \geq 1$ e $p+q \neq 3$, então um dos fechos das componentes é difeomorfo a $S^{p} \times D^{q+1}$

2) se $p+q=3$, isto é, $p=2$ e $q=1$, então um dos fechos das componentes é homeomorfo a $S^{2} \times D^{2}$.

\section{Conclusão}

O Teorema do toro de Alexander em [1, mostra que se temos um mergulho do toro $S^{1} \times S^{1}$ em $S^{3}$, então a imagem deste mergulho borda um toro sólido $S^{1} \times D^{2}$.

Neste trabalho, foi mostrado que para todo um mergulho de $S^{p} \times S^{q}$ em $S^{p+q+1}$, a imagem deste mergulho borda uma esfera cartesiano um disco, isto é, $S^{p} \times D^{q+1}$

O interessante é que não podemos generalizar esta idéia para mergulhos de $S^{p} \times S^{q} \times S^{r}$ em $S^{p+q+r+1}$. Em [18] é provado o seguinte;

Teorema 2.2.17. Seja $f: S^{p} \times S^{q} \times S^{r} \rightarrow S^{p+q+r+1}$ um mergulho suave com $2 \leq p \leq$ $q \leq r$. Suponhamos que $p+q \neq r$, ou $p+q=r$ e $r$ é par. Então o fecho de uma 
das componentes conexas por caminho de $S^{p+q+r+1}-f\left(S^{p} \times S^{q} \times S^{r}\right)$ é difeomorfo a $S^{p} \times S^{q} \times D^{r+1}$ ou $S^{p} \times D^{q+1} \times S^{r}$ ou $D^{p+1} \times S^{q} \times S^{r}$.

Por outro lado, quando $r$ é ímpar, é provado que;

Teorema 2.2.18. Se $p, q \geq 1$ e $p+q=r$ com $r$ impar, então existem infinitos mergulhos distintos $f_{n}: S^{p} \times S^{q} \times S^{r} \rightarrow S^{p+q+r+1}, n \in \mathbb{Z}-\{0\}$, tal que nenhum dos fechos das duas componentes conexas por caminhos de $S^{p+q+r+1}-f_{n}\left(S^{p} \times S^{q} \times S^{r}\right)$ é homotopicamente equivalente ao produto de duas esferas e um disco.

Os mergulhos do teorema 2.2.18 são chamados de mergulhos exóticos e são apresentados em [18]. 


\section{Referências Bibliográficas}

[1] Alexander, J. W., On the subdivision of a 3-space by a polyhedron, Proc. Nat. Acad. Sci. USA 10, 6-8, 1924.

[2] Boyer, S., Simply-connected 4-manifolds with a given boundary, Trans. Amer. Math. Soc., 298, 331-357, 1986.

[3] Bredon, G., Geometry and Topology, Springer, New York, 1993.

[4] Browder, W., Surgery on simple-connected manifolds, Ergebnisse der mathematik und ihrer grenzgebiete, Band 65, Springer-Verlag Berlin Heidelberg New York, 1972.

[5] Brown, M., A proof of the generalized Schoenflies theorem, Bull. Amer. Math. Soc., 66, 74-76, 1960.

[6] Dold, A., Lectures on Algebraic Topology, Springer-Verlag Berlin Heidelberg New York, 1980.

[7] Fenille, M. C., Mergulhos de produtos de três esferas e suas somas conexas em codimensão 1, Dissertação, ICMC, Universidade de São Paulo, São Carlos SP, Janeiro 2007.

[8] Greenberg, M. J., Harper, J. R.,Algebraic Topology - A First Course, Northeastern University, W. A. Benjamin, Inc. New York, 1967.

[9] Goldstein, R.Z., Piecewise linear unknotting of $S^{p} \times S^{q}$ in $S^{p+q+1}$, Michigan Math. J., 14 (1967), pg 405-415, MR 36 \#3365, Zbl 0157.54801. 
[10] Haefliger, A., Differentiable imbeddings, Bull. Amer. Math. Soc., 67, 109-112, 1961.

[11] Hirsch, M. W., Differential topology, Springer-Verlag, 1976.

[12] Kervaire, M., Normal bundle to a sphere in euclidean space, Bull. Amer. Math. Soc., 63, 147, 1957.

[13] Kosinski, A., On Alexander's theorem and knotted spheres, in 'Topology of 3Manifolds and Related Topics', ed. by M.K. Fort Jr., Prentice-Hall, (1961), pg 5557, MR $28 \# 1594$.

[14] Kosniowski, C., A first course in algebraic topology, Cambridge University Press, 1980.

[15] Lima, E. L., Grupo Fundamental e Espaços de Recobrimento, IMPA-Projeto Euclides, 2006.

[16] Lima, E. L., Homologia Básica, IMPA-Projeto Euclides, 2009.

[17] Lucas, L. A., Mergulhos em codimensão um de produtos cartesiano de três esferas, Tese, ICMC, Universidade de São Paulo, São Carlos SP, Junho 1998.

[18] Lucas, L. A., Saeki, O., Embeddings of $S^{p} \times S^{q} \times S^{r}$ in $S^{p+q+r+1}$, Pacific J. Math., $\mathbf{2 0 7}(2002)$, pg 447-462.

[19] Lucas, L. A., Saeki, O., Codimension one embeddings of product of three spheres, Topology Appl., 146-147 (2005), pg 409-419.

[20] Lucas, L. A., Neto, O. M., Saeki, O., A generalization os Alexander's torus theorem to hight dimensions and an unknotting theorem for $S^{p} \times S^{q}$ embedded in $S^{p+q+2}$, Kobe J. Math. 13, 145-165, 1996.

[21] Magnus, W., Karrass, A. and Solitar, D., "Combinatorial group theory: presentations of groups interms of generators and relations," second revised edition, Dover Publications Inc., New York, 1976. 
[22] Massey, W. S., On the normal bundle of a sphere imbedded in euclidean space, Proc. Amer. Math. Soc., 10, 959-964, 1959.

[23] Milnor, J., Lectures on the h-Cobordism Theorem, Math. Notes, Princeton University Press, Princeton, NJ, 1965.

[24] Munkres, J. R., Elements of Algebraic Topology, Westview Press, 1984.

[25] Rolfsen, D., Knots and Links, AMS Chelsea, 1976.

[26] Rourke, C. P., Sanderson, B.J., Introduction to Piecewise-Linear Topology, SpringerVerlag Berlin Heidelberg New York, 1972.

[27] Smale, S., On the structure of mennifolds Amer. J, Math., 84, 387-399, 1962.

[28] Smale, S., On the structure of 5-manifolds, Ann. of Math., 75, 38-46, 1962.

[29] Spanier, E. H., Algebraic Topology, Springer-Verlag Berlin Heidelberg New York, 1966.

[30] Steennrod, N., The topology of fibre bundles, Princeton University Press, 1951.

[31] Vick, J. W., Homology Theory-An Introduction to Algebraic Topology, SpringerVerlag Berlin Heidelberg New York, 1973.

[32] Wall, C. T. C., Diffeomorphisms of 4-manifolds, London Math. Soc., 39, 131-140, 1964.

[33] Wall, C. T. C., Unknotting tori in codimension one and spheres in codimension two, Proc. Camb. Phil. Soc., 61, 659-664, 1965.

[34] Whitehead, G. W., Elements of Homotopy Theory, Springer-Verlag Berlin Heidelberg New York, 1978.

[35] Whitney, H., The self-intersections of a smooth n-manifold in 2n-space, Ann. of Math., 45, 220-246, 1944. 